\title{
THE CONNECTION MATRIX THEORY FOR MORSE DECOMPOSITIONS
}

\author{
ROBERT.D. FRANZOSA
}

\begin{abstract}
The connection matrix theory for Morse decompositions is introduced. The connection matrices are matrices of maps between the homology indices of the sets in the Morse decomposition. The connection matrices cover, in a natural way, the homology index braid of the Morse decomposition and provide information about the structure of the Morse decomposition. The existence of connection matrices of Morse decompositions is established, and examples illustrating applications of the connection matrix are provided.
\end{abstract}

\section{INTRODUCTION}

In [4] the Conley index theory is extended to an index theory for partially ordered Morse decompositions of isolated invariant sets. Via an index filtration for the flow-defined ordering of the Morse decomposition the homology index braid of the Morse decomposition is defined. The homology index braid contains the homology of the Conley index of each Morse set, i.e., each isolated invariant set defined by the Morse decomposition, along with flow defined maps between these homology complexes. A connection matrix of the Morse decomposition is a matrix of maps between the homology indices of the minimal Morse sets which, in a natural way, defines an isomorphic image of the homology index braid. Thus, the connection matrices represent a simple codification of the information in the homology index braid. Since the homology index braid contains information about the structure of the invariant set and its Morse decomposition, so do the connection matrices. It is this feature of the connection matrices that is the main motivation for their study.

The connection matrix theory is presented by the author in his $\mathrm{Ph}$. D. thesis [3]. In [3] the theory is developed for the case where the homology of the Conley index is computed using field coefficients. In this paper we present the full connection matrix theory in the more general setting where the homology indices may be computed with coefficients in a module over a PID.

As the Conley index is a generalization of the classical Morse index, the connection matrix theory has correspondences within Morse theory. In that

Received by the editors September 18, 1986 and, in revised form, October 6, 1987.

1980 Mathematics Subject Classification (1985 Revision). Primary 58F35; Secondary 58F12, $34 \mathrm{C} 35$.

Key words and phrases. Conley index, Morse decomposition, connection matrix. 
setting the Leray spectral sequence is used to study the relationship between homology complexes associated to a decomposition of a set and the homology of the set. That work and the work on connection matrices are both motivated by a desire to obtain information about the structure of the sets defined by a decomposition from indices of minimal sets in the decomposition.

Applications of the connection matrix theory can be found in [11-13]. Further developments in the connection matrix theory can be found in [10], where mappings between flows and their relationship to connection matrices are studied, and in [6], where Rybakowski's Conley-index theory for semiflows on metric spaces is extended to include a connection matrix theory for Morse decompositions.

This paper begins in $\S 1$ with a summary of the main background results on homology index braids from [4]. $\S 1$ also includes a brief discussion motivating the work that follows. The connection matrix theory is developed on a purely algebraic level in the next three sections. In $\S \S 2$ and 3 the relevant algebraic structures are introduced (including the connection matrix in $\S 3$ ), and in $\S 4$ the main connection matrix existence result is proved. In $\S 5$ the connection matrix theory for Morse decompositions is discussed, and applications are presented in $\S 6$.

\section{BACKGROUND AND MOTIVATION}

The work in this paper is a continuation of that in [4], and therefore we carry over all of the notations and conventions used there. We summarize briefly the important definitions relating to partial orders, Morse decompositions, index filtrations and their associated chain complex braids, and homology index braids. Further details on these topics can be found in [4]. We assume that the reader is familiar with the basic concepts of the Conley index theory as in [1-4, 7-9, $14,15]$; in particular, flows, isolated invariant sets and isolating neighborhoods, $\omega$ and $\omega^{*}$ limit sets, attractor-repeller pairs, index pairs, index spaces, and the Conley index.

The standard reference to the homology theory used here is Spanier [16]. Unless otherwise indicated, the homology of a topological space is assumed to be singular homology with coefficients in a module over a PID. We point out that throughout this paper we frequently use the same symbol to denote a chain map and its induced homology map, rather than appending a subscript $*$ to denote the homology map.

Throughout this paper $P$ denotes a finite indexing set with $p$ elements. A partial order on $P$ is a relation, $<$, on the elements of $P$ satisfying:

(1) $\pi<\pi$ never holds for $\pi \in P$,

(2) $\pi<\pi^{\prime}$ and $\pi^{\prime}<\pi^{\prime \prime}$ imply $\pi<\pi^{\prime \prime}$.

Assume throughout that $<$ is a partial order on $P$.

An extension of $<$ is a partial order $<^{\prime}$ on $P$ for which $\pi<\pi^{\prime}$ implies $\pi<^{\prime} \pi^{\prime}$. If $P^{\prime} \subset P$, then $<$ induces a partial order on $P^{\prime}$ called the restriction of $<$ to $P^{\prime}$. 
An interval in $<$ is a subset $I \subset P$ for which $\pi, \pi^{\prime} \in I$ and $\pi<\pi^{\prime \prime}<\pi^{\prime}$ imply $\pi^{\prime \prime} \in I$. The set of intervals in $<$ is denoted $I(<) . I \in I(<)$ is called an attracting interval if $\pi \in I$ and $\pi^{\prime}<\pi$ imply $\pi^{\prime} \in I$. The set of attracting intervals in $<$ is denoted $A(<) . \pi, \pi^{\prime} \in P$ are called adjacent if $\left\{\pi, \pi^{\prime}\right\} \in I(<)$.

An adjacent $n$-tuple of intervals in $<$ is an ordered collection $\left(I_{1}, \ldots, I_{n}\right)$ of mutually disjoint intervals in < satisfying:

(1) $\bigcup_{i=1}^{n} I_{i} \in I(<)$,

(2) $\pi \in I_{j}, \pi^{\prime} \in I_{k}, j<k$ imply $\pi^{\prime} \nless \pi$.

The collection of adjacent $n$-tuples of intervals in $<$ is denoted $I_{n}(<)$. Note that $I(<)=I_{1}(<)$. It is easy to see that if $<^{\prime}$ is either an extension of $<$ or the restriction of $<$ to an interval in $<$ then $I_{n}\left(<^{\prime}\right) \subset I_{n}(<)$. If $(I, J)$ is an adjacent pair (i.e., 2-tuple) of intervals, then we set $I J=I \cup J$. If $(I, J)$ and $(J, I)$ are both adjacent pairs of intervals, then we say that $I$ and $J$ are noncomparable. If $\left(I_{1}, \ldots, I_{n}\right) \in I_{n}(<)$ and $\bigcup_{i=1}^{n} I_{i}=I$, then $\left(I_{1}, \ldots, I_{n}\right)$ is called a decomposition of $I$.

Throughout this paper assume $S$ is an isolated invariant set in $X$, a locally compact metric local flow in a Hausdorff topological space $\Gamma$ on which there is defined a flow.

If $S_{1}$ and $S_{2}$ are compact invariant subsets of $S$, then $C\left(S_{2}, S_{1}\right)$ is the set of orbits connecting $S_{2}$ to $S_{1}$ in $S$, i.e., the set $\left\{\gamma \in S \mid \omega(\gamma) \subset S_{1}\right.$ and $\left.\omega^{*}(\gamma) \subset S_{2}\right\}$. An attractor-repeller pair in $S,\left(A, A^{*}\right)$, decomposes $S$ into the union, $S=A \cup C\left(A^{*}, A\right) \cup A^{*}$. This idea is generalized via the Morse decompositions of $S$; specifically,

Definition 1.1. A (<-ordered) Morse decomposition of $S$ is a collection $M=$ $M(s)=\{\boldsymbol{M}(\pi)\}_{\pi \in P}$ of mutually disjoint compact invariant subsets of $S$ such that if $\gamma \in S \backslash \bigcup_{\pi \in P} M(\pi)$, then there exists $\pi<\pi^{\prime}$ with $\gamma \in C\left(M\left(\pi^{\prime}\right), M(\pi)\right)$.

Since a collection of sets $M=\{M(\pi)\}_{\pi \in P}$ can be a Morse decomposition of more than one invariant set, the structures (including the indices) associated to a Morse decomposition of an invariant set $S$ are defined relative to $S$. However, for notational convenience, we usually omit reference to $S$ in the discussions of the structures.

Assume for the remainder of the paper that $M=\{M(\pi)\}_{\pi \in P}$ is a <-ordered Morse decomposition of $S$. The partial order $<$ on $P$ induces an obvious partial order on $M$ called an admissible ordering of the Morse decomposition. The flow defines an "extremal" admissible ordering of $M$ called the flow ordering of $M$, denoted $<_{F}$ and such that $\pi<_{F} \pi^{\prime}$ if and only if there exists a sequence of distinct elements of $P: \pi=\pi_{0}, \ldots, \pi_{n}=\pi^{\prime}$ with $C\left(M\left(\pi_{j}\right), M\left(\pi_{j-1}\right)\right) \neq \varnothing$ for each $j=1, \ldots, n$. Every admissible ordering of $M$ is an extension of $<_{F}$.

Associated to the admissible ordering $<$ of $M$ there is a distinguished collection of subsets of $S$, called the Morse sets of $<$ and defined for each $I \in I(<)$ 
by

$$
M(I)=\left(\bigcup_{\pi \in I} M(\pi)\right) \cup\left(\bigcup_{\pi, \pi^{\prime} \in I} C\left(M\left(\pi^{\prime}\right), M(\pi)\right)\right) .
$$

$S$ being an isolated invariant set implies that each Morse set is too. Since every admissible ordering of $M$ is an extension of the flow ordering, it follows that the collection of Morse sets of the flow ordering contains the Morse sets of each other admissible ordering. If $(I, J) \in I_{2}(<)$, then $(M(I), M(J))$ is an attractor-repeller pair in $M(I J)$; in particular, if $I$ is an attracting interval in $<$, then $M(I)$ is an attractor in $S$ with complementary repeller $M(P \backslash I)$.

The index pair for an isolated invariant set is generalized by the index filtration for an admissible ordering of a Morse decomposition; specifically,

Definition 1.2. An index filtration for the admissible ordering $<$ of $M$ is a collection of compact sets $\mathscr{N}=\{N(I)\}_{I \in A(<)}$ satisfying:

(1) for each $I \in A(<),(N(I), N(\varnothing))$ is an index pair for the attractor $M(I)$,

(2) for each $I_{1}, I_{2} \in A(<), N\left(I_{1} \cap I_{2}\right)=N\left(I_{1}\right) \cap N\left(I_{2}\right)$ and $N\left(I_{1} \cup I_{2}\right)=$ $N\left(I_{1}\right) \cup N\left(I_{2}\right)$.

Assume throughout that $\mathcal{N}$ is an index filtration for the admissible ordering $<$ of $M$. If $J \in I(<)$ and $(I, J)$ is a decomposition of $K \in A(<)$, then it follows that $I \in A(<)$ and $(N(K), N(I))$ is an index pair for the Morse set $M(J)$. Thus the index filtration defines an index pair for each Morse set of the admissible ordering. Furthermore, it follows from property 2 in Definition 1.2 that if $\left(N\left(K_{i}\right), N\left(I_{i}\right)\right), i=1,2$, are index pairs for $M(J)$ defined by $\mathscr{N}$, then the index spaces $N\left(K_{i}\right) / N\left(I_{i}\right), i=1,2$, are homeomorphic. If we choose a coefficient module $G$, and let $C\left(N\left(K_{i}\right) / N\left(I_{i}\right) ; G\right)$ denote the singular chains of the index space $N\left(K_{i}\right) / N\left(I_{i}\right)$ with coefficients in $G$, then it follows that there is defined a chain complex $C_{\mathscr{N}}(J ; G)$ (also denoted $C(J)$ for simpler notation) which is naturally isomorphic to each $C\left(N\left(K_{i}\right) / N\left(I_{i}\right) ; G\right)$. Passing to homology in $C(J)$ one obtains $H_{*}(h(M(J)) ; G)$, the singular homology with coefficients in $G$ of $h(M(J))$, the Conley index of the Morse set $M(J)$. This is also called the homology index of $M(J)$ with coefficients in $G$, and for simplicity we denote it by $H(J)$.

Now if $(I, J) \in I_{2}(<)$ then chain maps are defined,

$$
C(I) \stackrel{i(I, I J)}{\longrightarrow} C(I J) \stackrel{p(I J, J)}{\longrightarrow} C(J)
$$

having the following properties:

(1) $i(I, I J)$ is injective and $p(I J, J) i(I, I J)=0$,

(2) the chain map defined by $p(I J, J), \rho: C(I J) / \operatorname{im}(i(I, I J)) \rightarrow C(J)$ induces an isomorphism on homology,

(3) if $I$ and $J$ are noncomparable, then $p(J I, I) i(I, I J)=\mathrm{id} \mid C(I)$,

(4) if $(I, J, K) \in I_{3}(<)$, then braid diagram (1.1), below, commutes. 


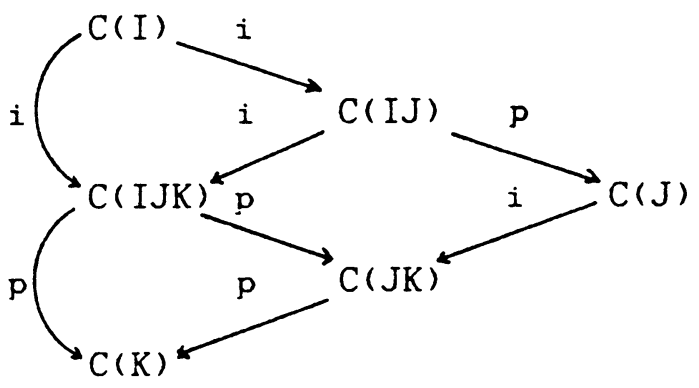

This collection of chain complexes and chain maps is called the chain complex braid of the index filtration with coefficients in $G$ and is denoted $\mathscr{C}(\mathscr{N} ; G)$ or $\mathscr{C}(\mathcal{N}) . \mathscr{C}(\mathcal{N})$ is introduced in [4] without mention of the injectivity of $i(I, I J)$. It is easy to see from the definition of $i(I, I J)$ in [4] that it is injective.

The chain complex braid of an index filtration is the model of what in $\S 2$ is more generally defined as a chain complex braid. Passing to homology in $\mathscr{C}(\mathscr{N})$ we obtain the homology index braid of the admissible ordering of the Morse decomposition with coefficients in $G$, denoted $\mathscr{H}(<; G)$ or $\mathscr{H}(<) . \mathscr{H}(<)$ is independent of the index filtration $\mathscr{N}$.

$\mathscr{H}(<)$ consists of graded modules $H(I)$ for each $I \in I(<)$, and maps between graded modules $i(I, I J): H(I) \rightarrow H(I J), p(I J, J): H(I J) \rightarrow H(J)$, and $\partial(J, I): H(J) \rightarrow H(I)$ satisfying:

(1) $\cdots \rightarrow H(I) \stackrel{i}{\rightarrow} H(I J) \stackrel{p}{\rightarrow} H(J) \stackrel{\overrightarrow{ }}{\rightarrow} H(I) \rightarrow \cdots$ is exact,

(2) if $I$ and $J$ are noncomparable, then $p(J I, I) i(I, I J)=\mathrm{id} \mid H(I)$,

(3) if $(I, J, K) \in I_{3}(<)$, then the following braid diagram (1.2) commutes:

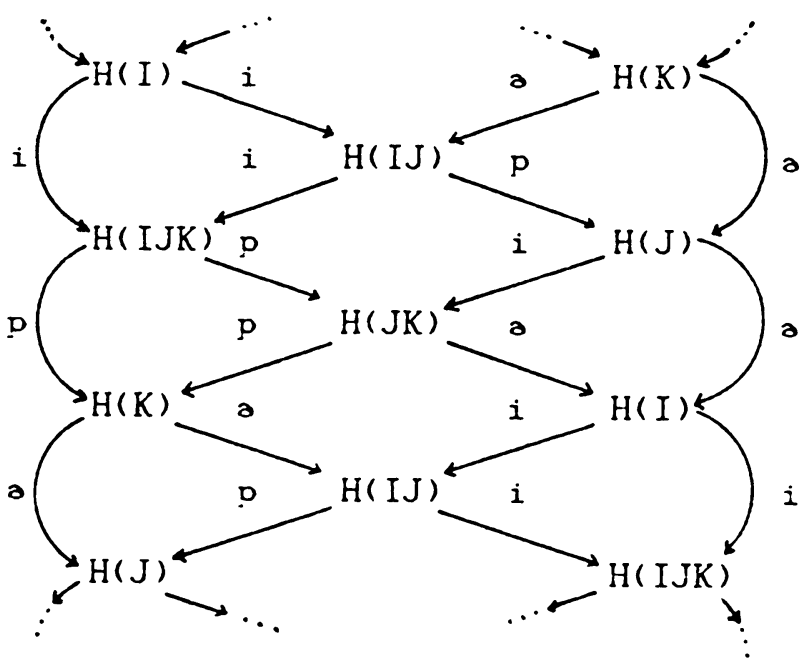


Because every admissible ordering of $M$ is an extension of the flow ordering, it follows that the homology index braid of the flow ordering contains the homology index braid of each other admissible ordering; therefore the homology index braid of the flow ordering is also called the homology index braid of the Morse decomposition and is denoted $\mathscr{H}(M ; G)$ or $\mathscr{H}(M)$.

The homology index braid is the model for what in $\S 2$ is more generally defined as a graded module braid. In $\S \S 3$ and 4 it is shown that the algebraic information in a graded module braid can be codified in a collection of matrices called the connection matrices of the graded module braid. In $\S 5$ the connection matrices of a Morse decomposition are then defined to be the connection matrices of the corresponding homology index braid. The definition and proof of existence of connection matrices is done on a purely algebraic level in $\S \S 2-4$.

Before commencing with the work on connection matrices consider the following simple case motivating their study.

Assume that $\left(A, A^{*}\right)$ is an attractor-repeller pair in $S$, and let $H(A), H\left(A^{*}\right)$, and $H(S)$ denote the homology indices with coefficients in a fixed field. The homology index braid in this case reduces to an exact sequence:

$$
\cdots \rightarrow H(A) \stackrel{i}{\rightarrow} H(S) \stackrel{p}{\rightarrow} H\left(A^{*}\right) \stackrel{\partial}{\rightarrow} H(A) \rightarrow \cdots .
$$

If $\partial \neq 0$, then $C\left(A^{*}, A\right) \neq \varnothing$ (see [4, 8]); therefore the map $\partial$ (and, more generally, the homology index braid) contains information about the structure of the attractor-repeller pair in $S$.

Now let $C \Delta(S)$ be the chain complex with graded module $H(A) \oplus H\left(A^{*}\right)$ and boundary map defined by the matrix

$$
\Delta=\left(\begin{array}{ll}
0 & \partial \\
0 & 0
\end{array}\right):\left(\begin{array}{c}
H(A) \\
H\left(A^{*}\right)
\end{array}\right) \rightarrow\left(\begin{array}{c}
H(A) \\
H\left(A^{*}\right)
\end{array}\right) .
$$

Upon appropriate restriction $C \Delta(S)$ and $\Delta$ define chain complexes $C \Delta(A)$ and $C \Delta\left(A^{*}\right)$ with boundary maps $\Delta(A)$ and $\Delta\left(A^{*}\right)$, respectively, where $C \Delta(A)=$ $H(A), C \Delta\left(A^{*}\right)=H\left(A^{*}\right)$, and the boundary maps $\Delta(A)$ and $\Delta\left(A^{*}\right)$ are trivial. Now a short exact sequence is defined,

$$
0 \rightarrow C \Delta(A) \stackrel{i}{\rightarrow} C \Delta(S) \stackrel{p}{\rightarrow} C \Delta\left(A^{*}\right) \rightarrow 0,
$$

where $i$ and $p$ are the obvious inclusion and projection maps, respectively. Passing to homology we obtain

$$
\cdots \rightarrow H \Delta(A) \stackrel{i}{\rightarrow} H \Delta(S) \stackrel{p}{\rightarrow} H \Delta\left(A^{*}\right) \stackrel{\bar{s}}{\rightarrow} H \Delta(A) \rightarrow \cdots .
$$

It easily follows that $H \Delta(A)=H(A), H \Delta\left(A^{*}\right)=H\left(A^{*}\right)$, and $\bar{\partial}=\partial$. Therefore we have defined the following commutative diagram of homology modules and maps:

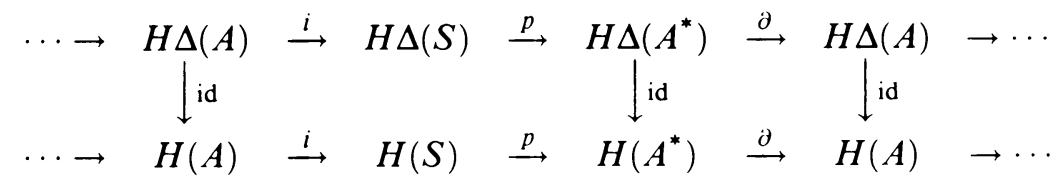


It is not difficult to see that there exist maps $\theta(S): H \Delta(S) \rightarrow H(S)$ making diagram (1.3) commute. The five lemma (see [16]) implies that such maps $\theta(S)$ are isomorphisms. Thus, via the matrix $\Delta$, an isomorphic image of the homology index braid is generated. One then expects that $\Delta$ contains information present in the homology index braid; in particular, in this case $\Delta$ contains $\partial$, and therefore provides information about the structure of the orbits connecting $A^{*}$ to $A$ in $S$. The matrix $\Delta$ is the prototype of the connection matrices studied in the following sections.

\section{GRADED MODULE BRAIDS AND CHAIN COMPLEX BRAIDS}

Definition 2.1. A sequence of chain maps

$$
C_{1} \stackrel{i}{\rightarrow} C_{2} \stackrel{p}{\rightarrow} C_{3}
$$

is called weakly exact if $i$ is injective, $p i=0$, and $\rho: C_{2} / \operatorname{im}(i) \rightarrow C_{3}$, the chain map defined by $p$, induces an isomorphism on homology.

Let $C_{1} \stackrel{i}{\rightarrow} C_{2} \stackrel{p}{\rightarrow} C_{3}$ be weakly exact, and denote the boundary map in $C_{i}$ by $\partial_{i}$ for each $i$. It is not difficult to see that if $\alpha \in H\left(C_{3}\right)$ and $X_{\alpha} \subset \operatorname{ker}\left(\partial_{3}\right)$ is the equivalence class defining $\alpha$, then $i^{-1} \partial_{2} p^{-1}\left(X_{\alpha}\right) \subset \operatorname{ker}\left(\partial_{1}\right)$ and represents a unique homology class in $H\left(C_{1}\right)$. Therefore, as with short exact sequences of chain complexes (see [16]), there exists a connecting homomorphism $\partial: H\left(C_{3}\right) \rightarrow H\left(C_{1}\right)$ and an associated exact homology sequence. Specifically,

Proposition 2.2. Given a weakly exact sequence of chain complexes

$$
C_{1} \stackrel{i}{\rightarrow} C_{2} \stackrel{p}{\rightarrow} C_{3}
$$

there exists a natural degree -1 homomorphism $\partial: H\left(C_{3}\right) \rightarrow H\left(C_{1}\right)$ such that:

(1) if $\alpha \in H\left(C_{3}\right)$ and $X_{\alpha} \subset \operatorname{ker}\left(\partial_{3}\right)$ is the equivalence class defining $\alpha$, then $\partial(\alpha)=\left[i^{-1} \partial_{2} p^{-1}\left(X_{\alpha}\right)\right]$,

(2) $\cdots \rightarrow H\left(C_{1}\right) \stackrel{i}{\rightarrow} H\left(C_{2}\right) \stackrel{p}{\rightarrow} H\left(C_{3}\right) \stackrel{\partial}{\rightarrow} H\left(C_{1}\right) \rightarrow \cdots$ is exact.

Proof. Let $\partial^{\prime}: H\left(C_{2} / \mathrm{im}(i)\right) \rightarrow H\left(C_{1}\right)$ be the connecting homomorphism for the short exact sequence of chain complexes

$$
0 \rightarrow C_{1} \stackrel{i}{\rightarrow} C_{2} \stackrel{p}{\rightarrow} C_{2} / \mathrm{im}(i) \rightarrow 0,
$$

where $p$ is projection onto the quotient, and let $\rho^{-1}$ be the inverse of the homology isomorphism $\rho: H\left(C_{2} / \operatorname{im}(i)\right) \rightarrow H\left(C_{3}\right)$. Then define $\partial: H\left(C_{3}\right) \rightarrow$ $H\left(C_{1}\right)$ by $\partial=\partial^{\prime} \rho^{-1}$. The naturality of $\partial$ and properties 1 and 2 are easily verified.

Definition 2.3. A graded module braid over $<$ is a collection $\mathscr{G}=\mathscr{G}(<)$ consisting of graded modules and maps between the graded modules satisfying:

(1) for each $I \in I(<)$ there is a graded module $G(I)$, 
(2) for each $(I, J) \in I_{2}(<)$ there are maps

$$
\begin{aligned}
& i(I, I J): G(I) \rightarrow G(I J) \text { of degree } 0, \\
& p(I J, J): G(I J) \rightarrow G(J) \text { of degree } 0, \\
& \partial(J, I): G(J) \rightarrow G(I) \text { of degree }-1
\end{aligned}
$$

which satisfy

(a) $\cdots \rightarrow G(I) \stackrel{i}{\rightarrow} G(I J) \stackrel{p}{\rightarrow} G(J) \stackrel{\partial}{\rightarrow} G(I) \rightarrow \cdots$ is exact,

(b) if $I$ and $J$ are noncomparable, then $p(J I, I) i(I, I J)=\mathrm{id} \mid G(I)$,

(c) if $(I, J, K) \in I_{3}(<)$, then the following braid diagram (called the $(I, J, K)$ braid diagram) commutes:

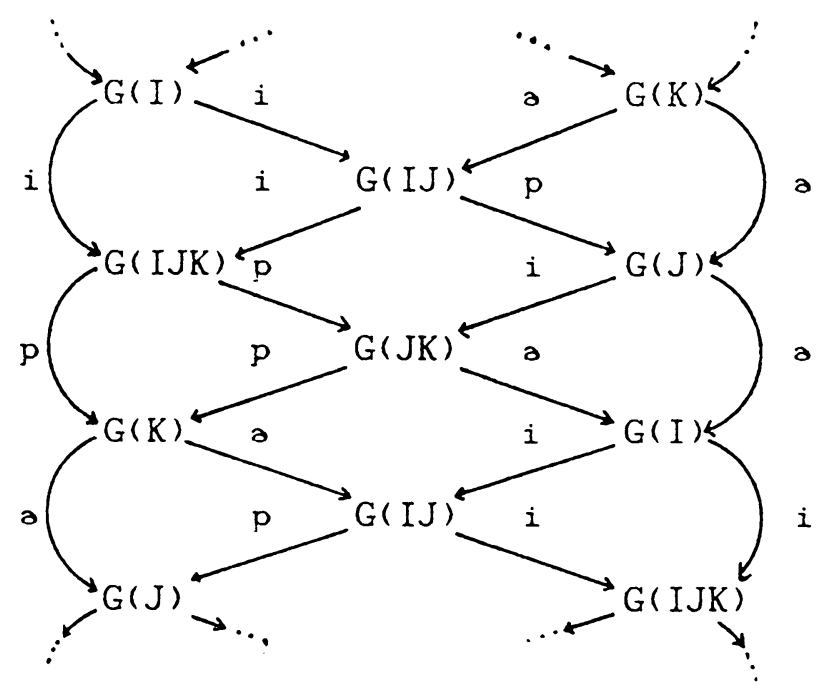

It is shown in [4] that $\mathscr{H}(<)$, the homology index braid of the admissible ordering < of $M$, is a graded module braid.

Assume that $\mathscr{G}$ and $\mathscr{G}^{\prime}$ are graded module braids over $<$.

Definition 2.4. A. A map $\theta$ between $\mathscr{G}$ and $\mathscr{G}^{\prime}$, denoted $\theta: \mathscr{G} \rightarrow \mathscr{G}^{\prime}$, is a collection of module homomorphisms $\theta(I): G(I) \rightarrow G(I), I \in I(<)$, such that for each $(I, J) \in I_{2}(<)$ the following diagram commutes:

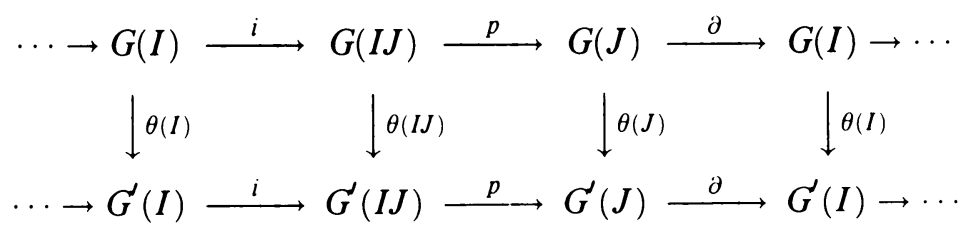

B. If $\theta(I)$ is an isomorphism for each $I \in I(<)$, then we call $\theta$ an isomorphism and we say that $\mathscr{G}$ and $\mathscr{G}^{\prime}$ are isomorphic. 
Proposition 2.5. Given $\theta: \mathscr{G} \rightarrow \mathscr{G}^{\prime}$. If $\theta(\pi)$ is an isomorphism for each $\pi \in P$ (i.e., for the one-element intervals), then $\theta$ is an isomorphism.

The proof of Proposition 2.5 is a straightforward induction argument using the five lemma.

If $\theta$ is an isomorphism, then there is an obvious inverse isomorphism, which we denote by $\theta^{-1}$, mapping $\mathscr{G}^{\prime}$ to $\mathscr{G}$.

Definition 2.6. A chain complex braid over $<$ is a collection $\mathscr{C}=\mathscr{C}(<)$ consisting of chain complexes and chain maps satisfying:

(1) for each $I \in I(<)$ there is a chain complex $C(I)$,

(2) for each $(I, J) \in I_{2}(<)$ there are chain maps

$$
i(I, I J): C(I) \rightarrow C(I J), \quad p(I J, J): C(I J) \rightarrow C(J)
$$

which satisfy:

(a) $C(I) \stackrel{i}{\rightarrow} C(I J) \stackrel{p}{\rightarrow} C(J)$ is weakly exact,

(b) if $I$ and $J$ are noncomparable, then $p(J I, I) i(I, I J)=\mathrm{id} \mid C(I)$,

(c) if $(I, J, K) \in I_{3}(<)$, then the following braid diagram (called the $(I, J, K)$ braid diagram) commutes:

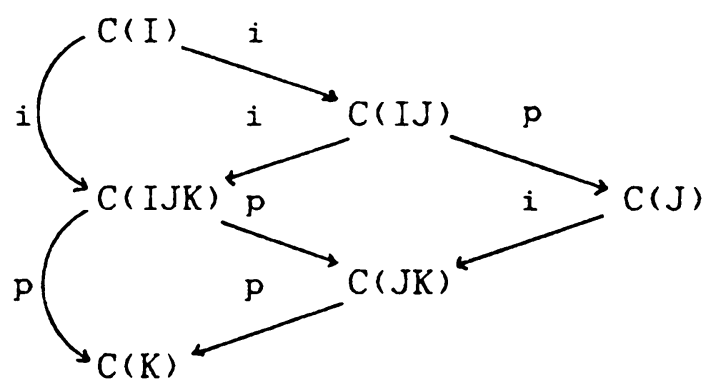

It is shown in [4] that $\mathscr{C}(\mathcal{N})$, the chain complex braid of the index filtration $\mathcal{N}$, is a chain complex braid.

Upon passing to homology, a chain complex braid defines a graded module braid. Specifically, assume $\mathscr{C}$ is a chain complex braid over $<$. For each $I \in$ $I(<)$ let $H(I)$ be the homology of the chain complex $C(I)$. If $(I, J) \in I_{2}(<)$, then there is a weakly exact sequence

$$
C(I) \stackrel{i(I, I J)}{\longrightarrow} C(I J) \stackrel{p(I J, J)}{\longrightarrow} C(J) .
$$

Associated to this weakly exact sequence there is an exact homology sequence

$$
\cdots \rightarrow H(I) \stackrel{i(I, I J)}{\longrightarrow} H(I J) \stackrel{p(I J, J)}{\longrightarrow} H(J) \stackrel{\partial(J, I)}{\longrightarrow} H(I) \rightarrow \cdots .
$$

Set $\mathscr{H} \mathscr{C}(<)$ equal to the collection consisting of the graded modules $H(I)$ for each $I \in I(<)$, along with the maps $i(I, I J), p(I J, J), \partial(J, I)$ from sequence $(2.3)$ for each $(I, J) \in I_{2}(<)$. 
Proposition 2.7. $\mathscr{H} \mathscr{C}(<)$ is a graded module braid over $<$.

Proof. The only property of a graded module braid required by Definition 2.3 that $\mathscr{H} \mathscr{C}(<)$ does not obviously satisfy is the commutativity of those parts of diagram (2.1) that contain a connecting boundary map $\partial$. We prove that diagram (2.4) below commutes for $(I, J, K) \in I_{3}(<)$. The other cases follow similarly.

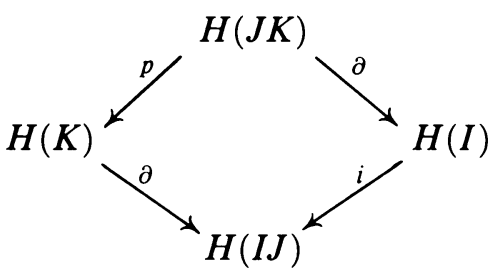

Consider

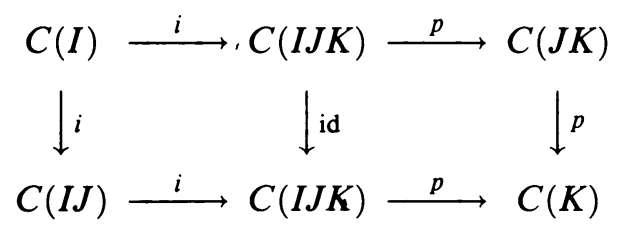

Since $\mathscr{C}(<)$ is a chain complex braid, it follows that diagram $(2.5)$ is a commutative diagram of chain maps with rows that are weakly exact. Upon passing to homology in diagram (2.5), it is easily seen that the commutativity of diagram (2.4) follows by the naturality of the connecting boundary map.

Definition 2.8. If $\mathscr{C}$ is a chain complex braid, then we call $\mathscr{H} \mathscr{C}$ the graded module braid generated by $\mathscr{C}$. Furthermore, if $\mathscr{G}$ is any graded module braid isomorphic to $\mathscr{H} \mathscr{C}$, then we say that $\mathscr{G}$ is chain complex generated.

It is shown in [4] that $\mathscr{H}(<)$, the homology index braid of the admissible ordering < of $M$, is the graded module braid generated by the chain complex braid $\mathscr{C}(\mathcal{N})$ where $\mathscr{N}$ is an index filtration for <.

The graded module braid is the input used in defining connection matrices. The chain complex braid is a support structure that is necessary in our proof of the existence of connection matrices.

Now assume that $\mathscr{C}$ and $\mathscr{C}^{\prime}$ are chain complex braids over $<$.

Definition 2.9. A chain map $\Psi$ between $\mathscr{C}$ and $\mathscr{C}^{\prime}$, denoted $\Psi: \mathscr{C} \rightarrow \mathscr{C}^{\prime}$, is a collection of chain maps $\Psi(I): C(I) \rightarrow C^{\prime}(I), I \in I(<)$, such that for each $(I, J) \in I_{2}(<)$ the following diagram commutes:

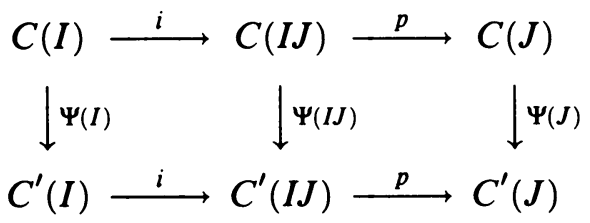

Now let $\mathscr{H} \mathscr{C}$ and $\mathscr{H} \mathscr{C}^{\prime}$ be the graded module braids generated by $\mathscr{C}$ and $\mathscr{C}^{\prime}$, respectively. Given a chain map $\Psi: \mathscr{C} \rightarrow \mathscr{C}^{\prime}$, by passing to homology we 
obtain a map $\theta: \mathscr{H} \mathscr{C} \rightarrow \mathscr{H} \mathscr{C}^{\prime}$, where for each $I \in I(<), \theta(I)$ is the map on homology induced by the chain map $\Psi(I)$. We call $\theta$ the homology map induced by $\Psi$.

Now, let $<$ be either an extension of $<$ or the restriction of $<$ to an interval $I \in I(<)$. Then $I_{n}\left(<^{\prime}\right) \subset I_{n}(<)$ for each $n$.

If $\mathscr{G}$ is a graded module braid over $<$, then we can restrict $\mathscr{G}$ to obtain a graded module braid $\mathscr{G} \mid<^{\prime}$ over $<^{\prime}$. Specifically, let $\mathscr{G} \mid<<^{\prime}$ be the collection consisting of the graded modules $G(I)$, for $I \in I\left(<^{\prime}\right) \subset I(<)$, along with the maps $i(I, I J), p(I J, J), \partial(J, I)$, for $(I, J) \in I_{2}\left(<^{\prime}\right) \subset I_{2}(<)$. It is not difficult to see that $\mathscr{G} \mid<^{\prime}$ is a graded module braid over $<^{\prime}$.

If $\theta: \mathscr{G} \rightarrow \mathscr{G}^{\prime}$ is a map between graded module braids over $<$, then we can restrict $\theta$ to a map

$$
\theta\left|<^{\prime}: \mathscr{G} K^{\prime} \rightarrow \mathscr{G}^{\prime} K^{\prime}, \quad \theta\right|^{\prime}=\left\{\theta(I) \mid I \in I\left(<^{\prime}\right)\right\} .
$$

We can similarly restrict chain complex braids over < and chain maps between chain complex braids. We leave the details to the reader.

\section{THE ALGEBRAIC CONNECTION MATRIX THEORY}

Let $C=\{C \Delta(\pi)\}_{\pi \in P}$ be a collection of graded modules. If $I \subset P$, then a map

$$
\Delta: \bigoplus_{\pi \in I} C \Delta(\pi) \rightarrow \bigoplus_{\pi \in I} C \Delta(\pi)
$$

can be regarded as a matrix

$$
\left(\begin{array}{c}
\vdots \\
\ldots \Delta_{\pi, \pi^{\prime}} \cdots \\
\vdots
\end{array}\right)_{\pi, \pi^{\prime} \in I}:\left(\begin{array}{c}
\vdots \\
C \Delta(\pi) \\
\vdots
\end{array}\right)_{\pi \in I} \rightarrow\left(\begin{array}{c}
\vdots \\
C \Delta(\pi) \\
\vdots
\end{array}\right)_{\pi \in I}
$$

where each $\Delta_{\pi, \pi^{\prime}}$ is a map from $C \Delta\left(\pi^{\prime}\right)$ to $C \Delta(\pi)$.

\section{Definition 3.1.}

A. $\Delta$ is upper triangular if $\Delta_{\pi, \pi^{\prime}} \neq 0$ implies $\pi \leq \pi^{\prime}$.

B. $\Delta$ is strictly upper triangular if $\Delta_{\pi, \pi^{\prime}} \neq 0$ implies $\pi<\pi^{\prime}$.

C. $\Delta$ is a boundary map if each $\Delta_{\pi, \pi^{\prime}}$ is of degree -1 and $\Delta^{2}=0$.

Now assume that $\Delta: \bigoplus_{\pi \in P} C \Delta(\pi) \rightarrow \bigoplus_{\pi \in P} C \Delta(\pi)$ is an upper triangular boundary map. For each $I \in I(<)$ set $C \Delta(I)=\bigoplus_{\pi \in I} C \Delta(\pi)$, and for $I$, $J \in I(<)$ let $\Delta(J, I): C \Delta(J) \rightarrow C \Delta(I)$ be the map defined by the matrix

$$
\left(\begin{array}{c}
\vdots \\
\cdots \Delta_{\pi, \pi^{\prime}} \cdots \\
\vdots
\end{array}\right)_{\pi \in I, \pi^{\prime} \in J}
$$

If $I \in I(<)$ then we denote $\Delta(I, I)$ by $\Delta(I)$. 
Proposition 3.2. $\Delta(I)$ is an upper triangular boundary map for each $I \in I(<)$. Proof. Clearly $\Delta(I)$ is upper triangular and $\Delta(I)_{\pi, \pi^{\prime}}$ is of degree -1 for each $\pi, \pi^{\prime} \in I$. We show that $\Delta(I)^{2}=0$. It is easy to see that there exist $H, J \in$ $I(<)$ such that $(H, I, J)$ is a decomposition of $P$ (i.e., $(H, I, J) \in I_{3}(<)$ and $H I J=P)$. Since $\Delta$ is upper triangular, $\Delta(H, I)=\Delta(H, J)=\Delta(I, J)=0$. We can view $\Delta$ as a map:

$$
\left(\begin{array}{ccc}
\Delta(H) & \Delta(I, H) & \Delta(J, H) \\
0 & \Delta(I) & \Delta(J, I) \\
0 & 0 & \Delta(J)
\end{array}\right):\left(\begin{array}{c}
C \Delta(H) \\
C \Delta(I) \\
C \Delta(J)
\end{array}\right) \rightarrow\left(\begin{array}{c}
C \Delta(H) \\
C \Delta(I) \\
C \Delta(J)
\end{array}\right) .
$$

$\Delta^{2}=0$; therefore by composing the middle row with the middle column it is easily seen that $\Delta(I)^{2}=0$.

Now for each $I \in I(<)$ there is a chain complex $C \Delta(I)$ with boundary map $\Delta(I)$. If $(I, J) \in I_{2}(<)$, then there is a short exact sequence

$$
0 \rightarrow C \Delta(I) \stackrel{i(I, I J)}{\longrightarrow} C \Delta(I J) \stackrel{p(I J, J)}{\longrightarrow} C \Delta(J) \rightarrow 0,
$$

where $i(I, I J)$ and $p(I J, J)$ are the obvious inclusion and projection maps, respectively.

Proposition 3.3. $i(I, I J)$ and $p(I J, J)$ are chain maps.

Proof. We can regard $i(I, I J)$ as a map of the form

$$
i(I, I J)=\left(\begin{array}{c}
\text { id } \\
0
\end{array}\right): C \Delta(I) \rightarrow\left(\begin{array}{c}
C \Delta(I) \\
C \Delta(J)
\end{array}\right) .
$$

As above we can view $\Delta(I J)$ as

$$
\left(\begin{array}{cc}
\Delta(I) & \Delta(J, I) \\
0 & \Delta(J)
\end{array}\right):\left(\begin{array}{l}
C \Delta(I) \\
C \Delta(J)
\end{array}\right) \rightarrow\left(\begin{array}{l}
C \Delta(I) \\
C \Delta(J)
\end{array}\right)
$$

With these identifications it follows that $i(I, I J) \Delta(I)=\Delta(J I) i(I, I J)$, and therefore $i(I, I J)$ is a chain map. The proof that $p(I J, J)$ is a chain map is similar.

It is not difficult to see that the collection of chain complexes and chain maps defined above satisfies the requirements in the definition of a chain complex braid. More specifically,

Proposition 3.4. Given an upper triangular boundary map

$$
\Delta: \bigoplus_{\pi \in P} C \Delta(\pi) \rightarrow \bigoplus_{\pi \in P} C \Delta(\pi)
$$

the collection, denoted $\mathscr{C} \Delta(<)$, consisting of the chain complexes $C \Delta(I)$ with boundary map $\Delta(I)$ for each $I \in I(<)$, and the chain maps $i(I, I J)$ and $p(I J, J)$ for each $(I, J) \in I_{2}(<)$, is a chain complex braid over $<$.

We call $\mathscr{C} \Delta(<)$ the chain complex braid defined by $\Delta$. Now let $\mathscr{H} \Delta(<)$ be the graded module braid generated by $\mathscr{C} \Delta(<)$; i.e., $\mathscr{H} \Delta(<)=\mathscr{H} \mathscr{C} \Delta(<)$. For 
each $I \in I(<)$ there is a graded module $H \Delta(I)$, and for each $(I, J) \in I_{2}(<)$ there is an exact sequence

$$
\cdots \rightarrow H \Delta(I) \stackrel{i(I, I J)}{\longrightarrow} H \Delta(I J) \stackrel{p(I J, J)}{\longrightarrow} H \Delta(J) \stackrel{\Delta(J, I)}{\longrightarrow} H \Delta(I) \rightarrow \cdots .
$$

The connecting homomorphism here takes on a particularly simple form. Specifically,

Proposition 3.5. If $[\alpha] \in H \Delta(J)$, then $\Delta(J, I)[\alpha]=[\Delta(J, I) \alpha]$.

The proof of Proposition 3.5 follows from Proposition 2.2.

Now given $\mathscr{G}$, a graded module braid over <, let $C=\{G(\pi)\}_{\pi \in P}$. It is natural to ask if there exist any upper triangular boundary maps

$$
\Delta: \bigoplus_{\pi \in P} G(\pi) \rightarrow \bigoplus_{\pi \in P} G(\pi)
$$

such that $\mathscr{H} \Delta$ is isomorphic to $\mathscr{G}$, and therefore if we can recover $\mathscr{G}$ from the collection of $G(\pi)$ 's via a matrix $\Delta$ (thereby using only the $G(I)$ 's for the one-element intervals). The significance of this is not so much in the recovering of $\mathscr{G}$, but rather in the codifying of the algebraic information in $\mathscr{G}$ via the matrix $\Delta$. In [3] it is shown that such maps $\Delta$ do exist if $\mathscr{G}$ is chain complex generated and $G(\pi)$ is free for each $\pi \in P$. Note that in this case if $G(\pi)$ is also finitely generated, then it follows that each diagonal entry $\Delta(\pi)$ in $\Delta$ must be trivial, and therefore $\Delta$ is strictly upper triangular.

If $G(\pi)$ is not free, then such a map $\Delta$ may not exist. As an example consider the graded module braid consisting of only the sequence of graded $\mathbb{Z}$-modules and maps

$$
\cdots \rightarrow G(2) \stackrel{\partial}{\longrightarrow} G(1) \stackrel{i}{\longrightarrow} G(12) \stackrel{p}{\longrightarrow} G(2) \stackrel{\partial}{\longrightarrow} G(1) \rightarrow \cdots,
$$

where the only nontrivial modules occur in dimension 1, and appear as follows:

$$
\cdots \rightarrow 0 \rightarrow \mathbb{Z} \stackrel{\times 2}{\longrightarrow} \mathbb{Z} \stackrel{p}{\longrightarrow} \mathbb{Z}_{2} \rightarrow 0 \rightarrow \cdots .
$$

It is easy to see that there is no upper triangular boundary map $\Delta: G(1) \oplus G(2) \rightarrow$ $G(1) \oplus G(2)$ resulting in homology isomorphic to $G(12)$.

This situation is overcome (in 3.8 below) by taking $C=\{C \Delta(\pi)\}_{\pi \in P}$, where each $C \Delta(\pi)$ is a free chain complex whose homology is isomorphic to $G(\pi)$.

Definition 3.6. Given $\mathscr{G}$, a graded module braid over $<$, and $C=\{C \Delta(\pi)\}_{\pi \in P}$, let $\Delta: \bigoplus_{\pi \in P} C \Delta(\pi) \rightarrow \bigoplus_{\pi \in P} C \Delta(\pi)$ be an upper triangular boundary map. Then

A. if $\mathscr{H} \Delta$ is isomorphic to $\mathscr{G}$, then $\Delta$ is called a $C$-connection matrix of $\mathscr{G}$,

B. if also $C=\{G(\pi)\}_{\pi \in P}$, then $\Delta$ is called a connection matrix of $\mathscr{G}$.

We denote the collection of $C$-connection matrices and connection matrices of $\mathscr{G}$ by $\mathscr{C} \mathscr{M}(\mathscr{G}, C)$ and $\mathscr{C} \mathscr{M}(\mathscr{G})$, respectively.

If $<^{\prime}$ is an extension of $<$, then $\mathscr{G} \mid<^{\prime} \subset \mathscr{G}$. Thus there are more algebraic restrictions in defining $\Delta$ for which $\mathscr{H} \Delta$ is isomorphic to $\mathscr{G}$ than in defining $\Delta$ for which $\mathscr{H} \Delta$ is isomorphic to $\mathscr{G} \mid<^{\prime}$. Thus we have the following proposition whose easy proof is left to the reader. 
Proposition 3.7. If $\mathscr{G}$ is a graded module braid over $<$, and $<$ is an extension of $<$, then for any $C, \mathscr{C} \mathscr{M}(\mathscr{G}, C) \subset \mathscr{C} \mathscr{M}\left(\mathscr{G} \mid<^{\prime}, C\right)$.

The following theorem, whose proof is presented in the next section, is the main connection matrix existence result.

Theorem 3.8. If $\mathscr{G}(<)$ is a chain complex generated graded module braid and $C=\{C \Delta(\pi)\}_{\pi \in P}$ is such that each $C \Delta(\pi)$ is a free chain complex with homology isomorphic to $G(\pi)$, then $\mathscr{C} \mathscr{M}(\mathscr{G}, C) \neq \varnothing$.

Note that if $\mathscr{G}$ is not chain complex generated, then there are no $(C$-) connection matrices $\Delta$ of $\mathscr{G}$; otherwise $\mathscr{G}$ would be isomorphic to $\mathscr{H} \Delta$, the graded module braid generated by $\mathscr{C} \Delta$, and therefore would be chain complex generated. It is, however, unknown if every graded module braid is chain complex generated, or equivalently, if there is a connection matrix for every graded module braid. This poses no problem for our application to Morse decompositions of invariant sets in a flow, because, as we have seen, the homology index braid of a Morse decomposition is a chain complex generated graded module braid, and therefore, we can associate connection matrices to each Morse decomposition.

It is interesting to note that a graded module braid does not necessarily have a unique connection matrix. In $\S 6$ we present an example that establishes the nonuniqueness of connection matrices and illustrates the significance of the nonuniqueness.

\section{THE EXISTENCE OF CONNECTION MATRICES}

We begin the proof of the existence of connection matrices by establishing some useful properties of chain complex braids. Assume $\mathscr{C}(<)$ is a chain complex braid over <.

Proposition 4.1. If $\left(I, J, J^{\prime}\right) \in I_{3}(<)$, and $J$ and $J^{\prime}$ are noncomparable, then the following triangle of chain maps is defined and commutes:

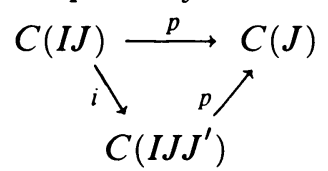

Proof. Note that $\left(I, J, J^{\prime}\right) \in I_{3}(<)$ and $J$ and $J^{\prime}$ noncomparable imply $\left(I, J^{\prime}, J\right) \in I_{3}(<)$. Applying $p\left(J^{\prime} J, J\right)$ to both sides of a commutativity relation derived from the $\left(I, J, J^{\prime}\right)$ braid diagram we obtain

$$
p\left(J^{\prime} J, J\right) i\left(J, J J^{\prime}\right) p(I J, J)=p\left(J^{\prime} J, J\right) p\left(I J J^{\prime}, J J^{\prime}\right) i\left(I J, I J J^{\prime}\right) .
$$

Noncomparability of $J$ and $J^{\prime}$ imply

$$
p\left(J^{\prime} J, J\right) i\left(J, J J^{\prime}\right)=\mathrm{id},
$$

and the $\left(I, J^{\prime}, J\right)$ braid diagram yields $p\left(J^{\prime} J, J\right) p\left(I J J^{\prime}, J J^{\prime}\right)=p\left(I J J^{\prime}, J\right)$. Thus

the desired relationship.

$$
p(I J, J)=p\left(I J J^{\prime}, J\right) i\left(I J, I J J^{\prime}\right),
$$


Proposition 4.2. If $\left(I_{1}, J_{1}\right)$ and $\left(I_{2}, J_{2}\right)$ are decompositions of an interval $K$, and $I_{2} \cap J_{1}=\varnothing$, then $p\left(K, J_{1}\right) i\left(I_{2}, K\right)=0$.

Proof. It is easy to see that $\left(I_{2}, I_{1} \cap J_{2}, J_{1}\right)$ is a decomposition of $K$. By weak exactness $p\left(K, J_{2}\right) i\left(I_{2}, K\right)=0$; thus $p\left(J_{2}, J_{1}\right) p\left(K, J_{2}\right) i\left(I_{2}, K\right)=0$. Via the $\left(I_{2}, I_{1} \cap J_{2}, J_{1}\right)$ braid diagram this reduces to $p\left(K, J_{1}\right) i\left(I_{2}, K\right)=0$, the desired relationship.

Now assume $\pi \in P$ is maximal under <. Set $A=A_{\pi}=\left\{\pi^{\prime} \in P \mid \pi^{\prime} \leq \pi\right\}$, and $R=R_{\pi}=P \backslash A$. If $K \in I(<)$ is such that $\pi \in K$, then we set $K_{A}=K \cap A$, $K_{R}=K \cap R$. Assume such a $K$ is fixed. The following proposition is a simple consequence of these definitions.

Proposition 4.3. $\left(K_{A}, K_{R}\right)$ and $\left(A \backslash K, K_{A}\right)$ are decompositions of $K$ and $A$ respectively.

Proposition 4.4. If $(I, J)$ is a decomposition of $K$, and $\pi \in I$, then $I_{A}=K_{A}$ and $\left(I_{A}, I_{R}, J\right) \in I_{3}(<)$.

Proof. Clearly $I_{A} \subset K_{A}$. If $\pi^{\prime} \in K_{A}$, then $\pi^{\prime} \leq \pi$. This and the fact that $\pi \in I$ imply that $\pi^{\prime} \in I$; thus $K_{A} \subset I_{A}$. Since $(I, J) \in I_{2}(<)$, and $\left(I_{A}, I_{R}\right)$ is a decomposition of $I$, it follows that $\left(I_{A}, I_{R}, J\right) \in I_{3}(<)$.

Proposition 4.5. If $(I, J)$ is a decomposition of $K$, and $\pi \in J$, then $\left(A \backslash K, K_{A} \backslash J_{A}, J_{A}\right),\left(K_{A}, K_{R} \backslash J_{R}, J_{R}\right),\left(I, J_{A}, J_{R}\right)$, and $\left(K_{A} \backslash J_{A}, J_{A}, K_{R} \backslash J_{R}\right)$ are adjacent triples of intervals, and $J_{A}$ and $K_{R} \backslash J_{R}$ are noncomparable.

Proof. $\left(A \backslash K, K_{A}\right) \in I_{2}(<)$ by Proposition 4.3, and it is easy to see that $\left(K_{A} \backslash J_{A}, J_{A}\right)$ is a decomposition of $K_{A}$; therefore $\left(A \backslash K, K_{A} \backslash J_{A}, J_{A}\right) \in I_{3}(<)$. $\left(K_{A}, K_{R}\right) \in I_{2}(<)$ by Proposition 4.3 , and $\left(K_{R} \backslash J_{R}, J_{R}\right)$ is clearly a decomposition of $K_{R}$; thus $\left(K_{A}, K_{R} \backslash J_{R}, J_{R}\right) \in I_{3}(<)$. Since $(I, J) \in I_{2}(<)$ and $\left(J_{A}, J_{R}\right)$ is a decomposition of $J$ (by Proposition 4.3), it follows that $\left(I, J_{A}, J_{R}\right) \in$ $I_{3}(<)$. It is not difficult to see that $\left(K_{A}, K_{R} \backslash J_{R}\right) \in I_{2}(<)$; this and the fact that $\left(K_{A} \backslash J_{A}, J_{A}\right)$ is a decomposition of $K_{A}$ imply $\left(K_{A} \backslash J_{A}, J_{A}, K_{R} \backslash J_{R}\right) \in I_{3}(<)$. Finally note that $J_{A} \subset A, K_{R} \backslash J_{R} \subset R$, and $(A, R) \in I_{2}(<)$, and furthermore $K_{R} \backslash J_{R} \subset I, J_{A} \subset J$, and $(I, J) \in I_{2}(<)$; it follows that $J_{A}$ and $K_{R} \backslash J_{R}$ are noncomparable.

Proposition 4.6. If $(I, J)$ is a decomposition of $K$, and $\pi \in J$, then the following diagram of maps is defined and commutes:

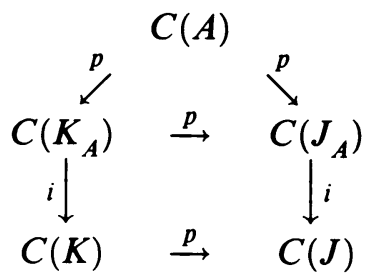


Proof. By Proposition 4.5, $\left(A \backslash K, K_{A} \backslash J_{A}, J_{A}\right) \in I_{3}(<)$; the associated braid diagram yields the commutative triangle. Now consider

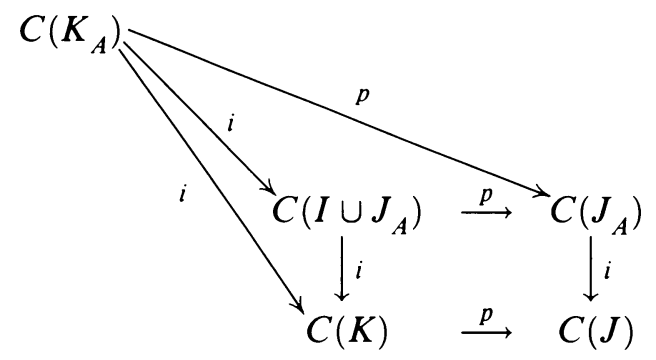

Note that $K_{A} \cup\left(K_{R} \backslash J_{R}\right)=I \cup J_{A}$. By Proposition 4.5, $\left(K_{A}, K_{R} \backslash J_{R}, J_{R}\right)$ and $\left(I, J_{A}, J_{R}\right)$ are adjacent triples of intervals, yielding, respectively, the left hand commutative triangle and the commutative rectangle in diagram (4.2). Furthermore, Proposition 4.5 states that $\left(K_{A} \backslash J_{A}, J_{A}, K_{R} \backslash J_{R}\right) \in I_{3}(<)$ and $J_{A}$ and $K_{R} \backslash J_{R}$ are noncomparable. Proposition 4.1 then yields the commutative triangle on the top in diagram (4.2). Thus the outside quadrilateral in diagram (4.2), i.e., the rectangle in diagram (4.1), commutes.

The following proposition describes the main construction step used in the proof of the existence of connection matrices.

Proposition 4.7. Consider the following diagram of chain complexes and chain maps:

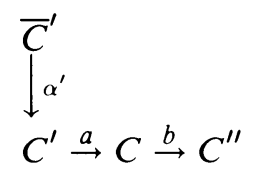

Assume that the horizontal sequence is weakly exact and that $\alpha^{\prime}$ induces an isomorphism on homology. If $\bar{C}^{\prime \prime}$ is a free chain complex with homology $\bar{H}^{\prime \prime}$ isomorphic to $H^{\prime \prime}$ (the homology of $C^{\prime \prime}$ ) via an isomorphism $\alpha^{\prime \prime}: \bar{H}^{\prime \prime} \rightarrow H^{\prime \prime}$, then there exist maps:

$$
d: \bar{C}^{\prime \prime} \rightarrow \bar{C}^{\prime}, \quad \tau: \bar{C}^{\prime \prime} \rightarrow C, \quad \alpha^{\prime \prime}: \bar{C}^{\prime \prime} \rightarrow C^{\prime \prime}
$$

such that

(1)

$$
\Delta:=\left(\begin{array}{cc}
\bar{\partial}^{\prime} & d \\
0 & \bar{\partial}^{\prime \prime}
\end{array}\right):\left(\begin{array}{c}
\bar{C}^{\prime} \\
\bar{C}^{\prime \prime}
\end{array}\right) \rightarrow\left(\begin{array}{c}
\bar{C}^{\prime} \\
\bar{C}^{\prime \prime}
\end{array}\right)
$$

is a boundary map, where $\bar{\partial}^{\prime}, \bar{\partial}^{\prime \prime}$ are the boundary maps in the chain complexes $\bar{C}^{\prime}, \bar{C}^{\prime \prime}$, respectively, $\alpha^{\prime \prime}$,

(2) $\alpha^{\prime \prime}: \bar{C}^{\prime \prime} \rightarrow C^{\prime \prime}$ is a chain map that induces the homology isomorphism

(3) $a \alpha^{\prime}+\tau: \bar{C}^{\prime} \oplus \bar{C}^{\prime \prime} \rightarrow C$ is a chain map, 
(4) the following diagram commutes:

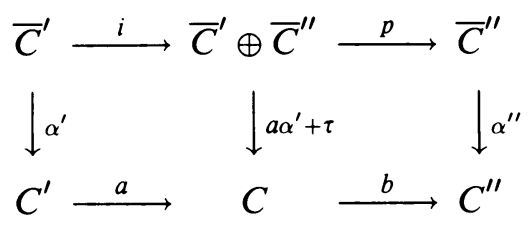

where $i, p$ are the obvious inclusion, projection maps respectively.

Proof. Set $K=\operatorname{ker}\left(\bar{\partial}^{\prime \prime}\right)$, and let $L$ be a subspace of $\bar{C}^{\prime \prime}$ complementary to $K$. We define $d, \tau$, and $\alpha^{\prime \prime}$ separately on $K$ and $L$. We begin by defining the maps on $K$ so that diagram (4.4) below commutes where the vertical maps on the front face are the boundary maps for the respective chain complexes, and the map $0: K \rightarrow K$ is the trivial map and takes the place of $d$ in $\Delta$.

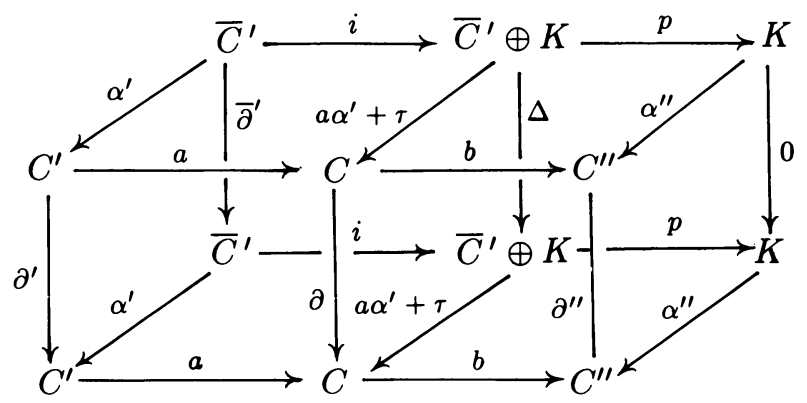

Let $\mathscr{k}$ be an element of a basis for $K$. Define $d(\not) \in \bar{C}^{\prime}$ so that $[d(\mathscr{k})] \in$ $\bar{H}^{\prime}$ is the image of $[\ell]$ under the following sequence of maps:

$$
\bar{H}^{\prime \prime} \stackrel{\alpha^{\prime \prime}}{\longrightarrow} H^{\prime \prime} \stackrel{\partial_{*}}{\longrightarrow} H^{\prime} \stackrel{\left(\alpha^{\prime}\right)^{-1}}{\longrightarrow} \bar{H}^{\prime}
$$

where $\partial_{*}: H^{\prime \prime} \rightarrow H^{\prime}$ is the connecting homomorphism for the weakly exact sequence in diagram (4.3) and $\left(\alpha^{\prime}\right)^{-1}$ is the inverse of the homology isomorphism induced by the chain map $\alpha^{\prime}$.

Now we claim that there exists $m \in C$ such that $b(m) \in \operatorname{ker}\left(\partial^{\prime \prime}\right),[b(m)]=$ $\alpha^{\prime \prime}([k])$, and $\partial(m)=a \alpha^{\prime} d(\mathscr{k})$. By Proposition 2.2 there exists $n \in C$ such that $[b(n)]=\alpha^{\prime \prime}([\ell])$ and $\left[a^{-1} \partial(n)\right]=\partial_{*} \alpha^{\prime \prime}([\ell])$. Then by the definition of $d(\mathscr{k})$ it follows that $\left[\alpha^{\prime} d(\mathscr{R})\right]=\left[a^{-1} \partial(n)\right]$. Choose $a \in a^{-1} \partial(n)$, and let $\mu \in C^{\prime}$ be such that $\partial^{\prime}(\mu)=\alpha^{\prime} d(\not)-a$. Set $m=n+a(\mu)$. Note that $b(m)=$ $b(n)+b a(\rho)=b(n)$; therefore $[b(m)]=[b(n)]=\alpha^{\prime \prime}([\ell])$. Furthermore, $\partial(m)=\partial(n)+a \partial^{\prime}(\mu)=\partial(n)+a \alpha^{\prime} d(k)-a(o)=\partial(n)+a \alpha^{\prime} d(\ell)-\partial(n)=$ $a \alpha^{\prime} d(\mathscr{K})$, completing the proof of the claim.

Now define $\tau(\mathscr{k})=m$ and $\alpha^{\prime \prime}(\mathscr{k})=b(m)$. With these definitions it is easy to see that diagram (4.4) commutes; therefore $d, \tau$, and $\alpha^{\prime \prime}$ are defined on $K$ as desired. Note furthermore that $\left[\alpha^{\prime \prime}(k)\right]=\alpha^{\prime \prime}[k]$; thus any extension 
of $\alpha^{\prime \prime}$ on $K$ to a chain map on $\bar{C}^{\prime \prime}$ yields a map that induces the homology isomorphism $\alpha^{\prime \prime}$.

The proof is complete once we define $d, \tau$, and $\alpha^{\prime \prime}$ on $L$ such that $\Delta^{2}=0$ and diagram (4.5) below commutes:

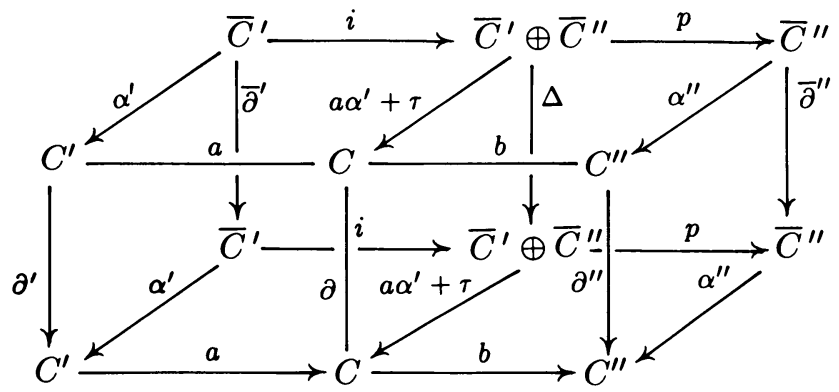

Let $\ell$ be an element of a basis for $L$. We define $d(\ell), \tau(\ell)$, and $\alpha^{\prime \prime}(\ell)$. Note that $\bar{\partial}^{\prime \prime}(\ell) \in K$; so $d \bar{\partial}^{\prime \prime}(\ell), \tau \bar{\partial}^{\prime \prime}(\ell)$, and $\alpha^{\prime \prime} \bar{\partial}^{\prime \prime}(\ell)$ are all defined. We claim that there exist $q \in \bar{C}^{\prime}, r \in C$ such that $\partial(\mu)=a \alpha^{\prime}(q)+\tau \bar{\partial}^{\prime \prime}(\ell)$. Since $\left[\bar{\partial}^{\prime \prime}(\ell)\right]=0$, it follows (by the definition of $d$ on $K$ ) that $\left[d \bar{\partial}^{\prime \prime}(\ell)\right]=0$ and there exists $\triangleleft \in \bar{C}^{\prime}$ such that $\bar{\partial}^{\prime}(\varsigma)=d \bar{\partial}^{\prime \prime}(\ell)$. Now

$$
\begin{aligned}
\left.\partial\left(a \alpha^{\prime}(-\lrcorner\right)+\tau \bar{\partial}^{\prime \prime}(\ell)\right) & \left.=\partial\left(a \alpha^{\prime}+\tau\right)(-\lrcorner \oplus \bar{\partial}^{\prime \prime}(\ell)\right) \\
& \left.=\left(a \alpha^{\prime}+\tau\right) \Delta(-\lrcorner \oplus \bar{\partial}^{\prime \prime}(\ell)\right) \\
& =\left(a \alpha^{\prime}+\tau\right)\left(\left(\bar{\partial}^{\prime}(-\jmath)+d \bar{\partial}^{\prime \prime}(\ell)\right) \oplus 0\right) \\
& =0,
\end{aligned}
$$

where the second equality holds by the commutativity of diagram (4.4), and the fourth equality holds because $\bar{\partial}^{\prime}(\jmath)=d \bar{\partial}^{\prime \prime}(\ell)$. It follows that $\left[a \alpha^{\prime}(-\jmath)+\right.$ $\left.\tau \bar{\partial}^{\prime \prime}(\ell)\right] \in H$, the homology of $C$, and

$$
b\left[a \alpha^{\prime}(-\varsigma)+\tau \bar{\partial}^{\prime \prime}(\ell)\right]=\left[b \tau \bar{\partial}^{\prime \prime}(\ell)\right]=\left[\alpha^{\prime \prime} \bar{\partial}^{\prime \prime}(\ell)\right]=\alpha^{\prime \prime}\left[\bar{\partial}^{\prime \prime}(\ell)\right]=0,
$$

where the second equality holds by the commutativity of diagram (4.4), and the fourth holds because $\left[\bar{\partial}^{\prime \prime}(\ell)\right]=0$. Therefore $\left.\left[a \alpha^{\prime}(-\lrcorner\right)+\tau \bar{\partial}^{\prime \prime}(\ell)\right]$ is in the image of the homology map, $a: H^{\prime} \rightarrow H$, induced by the chain map $a$, and furthermore, since $\alpha^{\prime}: \bar{H}^{\prime} \rightarrow H^{\prime}$ is an isomorphism, there exists $t \in \operatorname{ker}\left(\bar{\partial}^{\prime}\right)$ such that $a \alpha^{\prime}[t]=\left[a \alpha^{\prime}(-\delta)+\tau \bar{\partial}^{\prime \prime}(\ell)\right]$. Thus there exists $\mu \in C$ such that $\partial(\mu)=a \alpha^{\prime}(-\jmath)+\tau \bar{\partial}^{\prime \prime}(\ell)-a \alpha^{\prime}(t)$. Set $q=-\jmath-t$. Clearly $\partial(\mu)=a \alpha^{\prime}(q)+$ $\tau \bar{\partial}^{\prime \prime}(\ell)$; thus the proof of the claim is complete.

Now define $d(\ell)=q, \tau(\ell)=\mu$, and $\alpha^{\prime \prime}(\ell)=b(\mu)$.

We claim that $\Delta^{2}=0$. Clearly if $c \oplus 0 \in \bar{C}^{\prime} \oplus \bar{C}^{\prime \prime}$, then $\Delta^{2}(c \oplus 0)=0$. Also, if $0 \oplus \mathscr{K} \in \bar{C}^{\prime}$ with $\ell \in K$, then because $d(\mathscr{k}) \in \operatorname{ker}\left(\bar{\partial}^{\prime}\right)$, it follows that 
$\Delta^{2}(0 \oplus \ell)=0$. Consider $0 \oplus \ell \in \bar{C}^{\prime} \oplus \bar{C}^{\prime \prime}$ with $\ell \in L$.

$$
\begin{aligned}
\Delta^{2}(0 \oplus \ell) & =\Delta\left(d(\ell) \oplus \bar{\partial}^{\prime \prime}(\ell)\right) \\
& =\left(\bar{\partial}^{\prime} d(\ell)+d \bar{\partial}^{\prime \prime}(\ell)\right) \oplus \bar{\partial}^{\prime \prime} \bar{\partial}^{\prime \prime}(\ell) \\
& =\left(\bar{\partial}^{\prime}(-\jmath)+\bar{\partial}^{\prime}(-t)+d \bar{\partial}^{\prime \prime}(\ell)\right) \oplus 0 \\
& =0,
\end{aligned}
$$

where the last equality holds because $t \in \operatorname{ker}\left(\bar{\partial}^{\prime}\right)$ and $\bar{\partial}^{\prime}(\jmath)=d \bar{\partial}^{\prime \prime}(\ell)$.

Now we claim that diagram (4.5) commutes. The left and right faces on the right-hand cube need to be checked. That the other squares commute follows either by assumption (e.g., $a$ is a chain map) or directly from the definition of the particular maps. Thus we need to show that $a \alpha^{\prime}+\tau$ and $\alpha^{\prime \prime}$ are chain maps. That $\alpha^{\prime \prime}$ is a chain map, i.e., that the right face commutes, follows by surjectivity of $p$ and the commutativity of all of the other faces in the righthand cube once it is shown that $a \alpha^{\prime}+\tau$ is a chain map.

To prove that $a \alpha^{\prime}+\tau$ is a chain map we consider $\partial\left(a \alpha^{\prime}+\tau\right)$ and $\left(a \alpha^{\prime}+\tau\right) \Delta$ on $c \oplus 0,0 \oplus \ell$, and $0 \oplus \ell \in \bar{C}^{\prime} \oplus \bar{C}^{\prime \prime}$ as above. First consider $c \oplus 0$; $\partial\left(a \alpha^{\prime}+\tau\right)(c \oplus 0)=\partial a \alpha^{\prime}(c)=a \alpha^{\prime} \bar{\partial}^{\prime}(c)=\left(a \alpha^{\prime}+\tau\right) \Delta(c \oplus 0)$. Next consider $0 \oplus \mathscr{k} ; \partial\left(a \alpha^{\prime}+\tau\right)(0 \oplus \mathscr{k})=\partial \tau(\mathscr{k})=\partial(m)=a \alpha^{\prime} d(\not{k})=\left(a \alpha^{\prime}+\tau\right) \Delta(0 \oplus \mathscr{k})$, where the last equality holds because $\bar{\partial}^{\prime \prime}(\mathscr{C})=0$. Finally consider $0 \oplus \ell$; $\partial\left(a \alpha^{\prime}+\tau\right)(0 \oplus \ell)=\partial \tau(\ell)=\partial(\mu)=a \alpha^{\prime}(q)+\tau \bar{\partial}^{\prime \prime}(\ell)=a \alpha^{\prime} d(\ell)+\tau \bar{\partial}^{\prime \prime}(\ell)=$ $\left(a \alpha^{\prime}+\tau\right) \Delta(0 \oplus \ell)$. Thus $a \alpha^{\prime}+\tau$ is a chain map, and the proof of Proposition 4.7 is complete.

We leave it to the reader to provide the simple verification that the main connection matrix existence result, Theorem 3.8, is established via the following

Theorem 4.8. Let $\mathscr{C}(<)$ be a chain complex braid, and $C=\{C \Delta(\pi)\}_{\pi \in P}$ be such that for each $\pi \in P, C \Delta(\pi)$ is a free chain complex with homology $H \Delta(\pi)$ isomorphic to $H(\pi)$, the homology of the chain complex $C(\pi)$ in $\mathscr{C}(<)$. Then there exists an upper triangular boundary map,

$$
\Delta: \bigoplus_{\pi \in P} C \Delta(\pi) \rightarrow \bigoplus_{\pi \in P} C \Delta(\pi),
$$

and a chain map, $\Psi: \mathscr{C} \Delta(<) \rightarrow \mathscr{C}(<)$, from the chain complex braid defined by $\Delta$ to $\mathscr{C}(<)$, such that $\theta: \mathscr{H} \Delta(<) \rightarrow \mathscr{H} \mathscr{C}(<)$, the map induced by $\Psi$, is an isomorphism.

Proof. The proof is by induction on the order of the indexing set $P$. If $P$ contains one element $\pi$, then let $\Delta$ be the boundary map in the chain complex $C \Delta(\pi)$ and $\theta: H \Delta(\pi) \rightarrow H(\pi)$ be an isomorphism. Since $C \Delta(\pi)$ is free, there exists a chain map $\Psi: C \Delta(\pi) \rightarrow C(\pi)$ inducing $\theta$. Clearly $\Delta$ and $\Psi$ satisfy the requirements of the theorem.

Now assume the theorem is true for indexing sets of order $n-1$ and $P$ is of order $n$. 
Choose a maximal (under $<$ ) $\pi \in P$. Let $P^{\prime}=P \backslash \pi$ and $<^{\prime}$ be the restriction of $<$ to $P$. By induction there exists an upper triangular boundary map $\Delta^{\prime}: \bigoplus_{\pi \in P^{\prime}} C \Delta(\pi) \rightarrow \bigoplus_{\pi \in P^{\prime}} C \Delta(\pi)$ and a chain map between chain complex braids, $\Psi^{\prime}: \mathscr{C} \Delta^{\prime} \rightarrow \mathscr{C} \mid<^{\prime}$, that induces an isomorphism on homology.

We extend $\Delta^{\prime}$ to an upper triangular boundary map,

$$
\Delta: \bigoplus_{\pi \in P} C \Delta(\pi) \rightarrow \bigoplus_{\pi \in P} C \Delta(\pi)
$$

and $\Psi^{\prime}$ to a chain map, $\Psi: \mathscr{C} \Delta \rightarrow \mathscr{C}$, inducing an isomorphism on homology.

Since $\Delta$ is an extension of $\Delta^{\prime}$, we define $\Delta\left(P^{\prime}\right)=\Delta^{\prime} . \Delta$ must be upper triangular; therefore $\Delta\left(P^{\prime}, \pi\right)$ is defined to be zero.

It remains to define $\Delta(\pi, P)$; this is done in three separate pieces. Let $A=$ $\{\alpha \in P \mid \alpha \leq \pi\}$, and set $A^{\prime}=A \backslash \pi, R=P \backslash A .\left(A^{\prime}, R, \pi\right),\left(A^{\prime}, \pi, R\right) \in I_{3}(<)$. Upper triangularity of $\Delta$ requires the definition $\Delta(\pi, R)=0$. Set $\Delta(\pi)$ equal to the boundary map for the chain complex $C \Delta(\pi)$, and let $\theta(\pi): H \Delta(\pi) \rightarrow H(\pi)$ be an isomorphism. To define $\Delta\left(\pi, A^{\prime}\right)$ consider

$$
\begin{aligned}
& C \Delta\left(A^{\prime}\right) \\
& \quad\left\lfloor\Psi\left(A^{\prime}\right)\right. \\
& C\left(A^{\prime}\right) \stackrel{i\left(A^{\prime}, A\right)}{\longrightarrow} C(A) \stackrel{p(A, \pi)}{\longrightarrow} C(\pi) .
\end{aligned}
$$

The horizontal sequence is weakly exact, and by induction $\Psi\left(A^{\prime}\right)$ induces a homology map $\theta\left(A^{\prime}\right)$ which is an isomorphism. $C \Delta(\pi)$ is a free chain complex and $\theta(\pi): H \Delta(\pi) \rightarrow H(\pi)$ is an isomorphism.

By Proposition 4.7 there exists maps

$$
\begin{aligned}
& \Delta\left(\pi, A^{\prime}\right): C \Delta(\pi) \rightarrow C \Delta\left(A^{\prime}\right), \\
& \tau_{A}: C \Delta(\pi) \rightarrow C(A), \\
& \Psi(\pi): C \Delta(\pi) \rightarrow C(\pi)
\end{aligned}
$$

such that

(1)

$$
\Delta(A)=\left(\begin{array}{cc}
\Delta\left(A^{\prime}\right) & \Delta\left(\pi, A^{\prime}\right) \\
0 & \Delta(\pi)
\end{array}\right):\left(\begin{array}{c}
C \Delta\left(A^{\prime}\right) \\
C \Delta(\pi)
\end{array}\right) \rightarrow\left(\begin{array}{c}
C \Delta\left(A^{\prime}\right) \\
C \Delta(\pi)
\end{array}\right)
$$

is a boundary map,

(2) $\Psi(\pi)$ is a chain map that induces $\theta(\pi)$,

(3) $\tau_{A}+i \Psi\left(A^{\prime}\right): C \Delta(\pi) \oplus C \Delta\left(A^{\prime}\right) \rightarrow C(A)$ is a chain map,

(4) the following diagram commutes:

$$
\begin{aligned}
& C \Delta\left(A^{\prime}\right) \stackrel{i}{\longrightarrow} C \Delta\left(A^{\prime}\right) \oplus C \Delta(\pi) \stackrel{p}{\longrightarrow} C \Delta(\pi) \\
& \downarrow \Psi\left(A^{\prime}\right) \quad \downarrow_{\tau_{1}+i \Psi\left(A^{\prime}\right)}\lfloor\Psi(\pi) \\
& C\left(A^{\prime}\right) \stackrel{i}{\longrightarrow} C(A) \stackrel{p}{\longrightarrow} C(\pi)
\end{aligned}
$$


Now with this definition of $\Delta\left(\pi, A^{\prime}\right)$ the matrix $\Delta$ is completely defined. $\Delta$ is obviously upper triangular; we claim it is a boundary map. We can view $\Delta$ as a matrix

$$
\Delta=\left(\begin{array}{ccc}
\Delta\left(A^{\prime}\right) & \Delta\left(\pi, A^{\prime}\right) & \Delta\left(R, A^{\prime}\right) \\
0 & \Delta(\pi) & 0 \\
0 & 0 & \Delta(R)
\end{array}\right) .
$$

Then

$$
\Delta^{2}=\left(\begin{array}{ccc}
\Delta\left(A^{\prime}\right)^{2} & \Delta\left(A^{\prime}\right) \Delta\left(\pi, A^{\prime}\right)+\Delta\left(\pi, A^{\prime}\right) \Delta(\pi) & \Delta\left(A^{\prime}\right) \Delta\left(R, A^{\prime}\right)+\Delta\left(R, A^{\prime}\right) \Delta(R) \\
0 & \Delta(\pi)^{2} & 0 \\
0 & 0 & \Delta(R)^{2}
\end{array}\right) .
$$

Since $\left(\begin{array}{cc}\Delta\left(A^{\prime}\right) & \Delta\left(\pi, A^{\prime}\right) \\ 0 & \Delta(\pi)\end{array}\right)$ is a boundary map, it follows that $\Delta(\pi)^{2}, \Delta\left(A^{\prime}\right)^{2}$, and $\Delta\left(A^{\prime}\right) \Delta\left(\pi, A^{\prime}\right)+\Delta\left(\pi, A^{\prime}\right) \Delta(\pi)$ are zero. $\Delta\left(P^{\prime}\right)=\left(\begin{array}{c}\Delta\left(A^{\prime}\right) \Delta\left(R, A^{\prime}\right) \\ 0\end{array} \frac{\Delta(R)}{\Delta(R)}\right)$, and by induction $\Delta\left(P^{\prime}\right)^{2}=0$; therefore both $\Delta(R)^{2}$ and $\Delta\left(A^{\prime}\right) \Delta\left(R, A^{\prime}\right)+\Delta\left(R, A^{\prime}\right) \Delta(R)$ are zero. Hence $\Delta^{2}=0$ and it follows that $\Delta$ is an upper triangular boundary map.

We now define $\Psi: \mathscr{C} \Delta \rightarrow \mathscr{C}$. Since $\Psi$ is an extension of $\Psi^{\prime}$, we define $\Psi \mid<^{\prime}=\Psi^{\prime}$. It remains to define $\Psi(K)$ for intervals $K$ containing $\pi$. Set $K^{\prime}=K \backslash \pi$, and as in Propositions 4.3-4.6 with $A=A_{\pi}$ and $R=R_{\pi}$, let $K_{A}=K \cap A$ and $K_{R}=K \cap R$.

Note that Proposition 4.3 implies that both $p\left(A, K_{A}\right)$ and $i\left(K_{A}, K\right)$ are defined.

Define $\tau_{K}: C \Delta(\pi) \rightarrow C(K)$ to be the composition of the maps

$$
C \Delta(\pi) \stackrel{\tau_{A}}{\longrightarrow} C(A) \stackrel{p\left(A, K_{A}\right)}{\longrightarrow} C\left(K_{A}\right) \stackrel{i\left(K_{A}, K\right)}{\longrightarrow} C(K),
$$

and $\Psi(K): C \Delta(K) \rightarrow C(K)$ to be equal to the map

$$
\tau_{K}+i\left(K^{\prime}, K\right) \Psi\left(K^{\prime}\right): C \Delta(\pi) \oplus C \Delta\left(K^{\prime}\right) \rightarrow C(K) .
$$

Note that $\Psi(\pi)$ is defined twice; however, the definition of $\tau_{\pi}$ and the commutativity of diagram (4.6) imply that the two definitions coincide.

Now $\Psi$ is completely defined. To complete the proof of the theorem we must show that $\Psi$ is a chain map. Then, since $\Psi(\pi)$ induces an isomorphism on homology for each $\pi \in P$, it follows (by Proposition 2.5) that $\Psi$ induces an isomorphism on homology. Since by induction $\Psi \mid<^{\prime}$ is a chain map, we only need to prove that if $K \in I(<)$ and $\pi \in K$, then $\Psi(K)$ is a chain map, and furthermore, if $(I, J)$ is a decomposition of $K$, then the following diagram commutes:

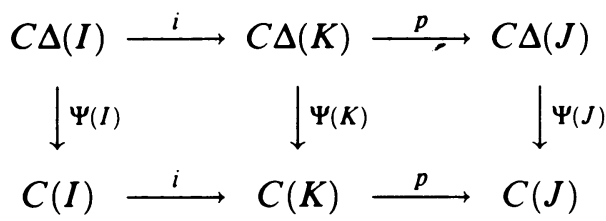

We first prove that diagram (4.7) commutes. We call the left-hand square in diagram (4.7) an inclusion square and the right-hand square a projection square. Each square is considered separately. 
To prove that the inclusion square commutes we consider two cases: $\pi \notin I$ and $\pi \in I$. Assume $\pi \notin I .(I, J / \pi, \pi) \in I_{3}(<)$. Consider

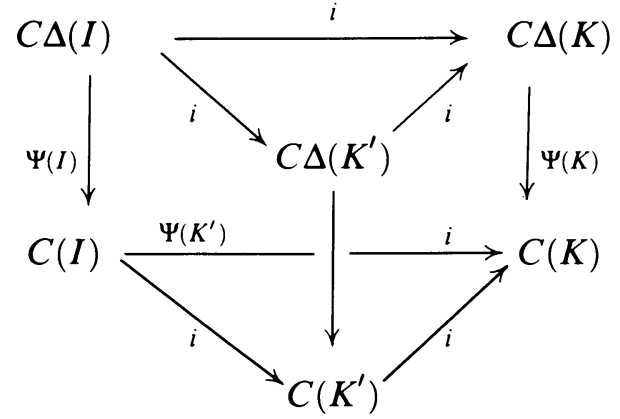

The top and bottom commutes by the $(I, J \backslash \pi, \pi)$ braid diagrams. The left side commutes by induction. The right side commutes by the definition of $\Psi(K)$. Therefore the back commutes; i.e., if $\pi \notin I$, then the inclusion square commutes.

Now assume $\pi \in I$. Set $I^{\prime}=I \backslash \pi . C \Delta(I)=C \Delta(\pi) \oplus C \Delta\left(I^{\prime}\right)$. We show that the inclusion square commutes on each of the subspaces $C \Delta(\pi)$ and $C \Delta\left(I^{\prime}\right)$ of $C \Delta(I)$. Note that $\left(I^{\prime}, \pi, J\right),\left(I^{\prime}, J, \pi\right) \in I_{3}(<)$. Consider

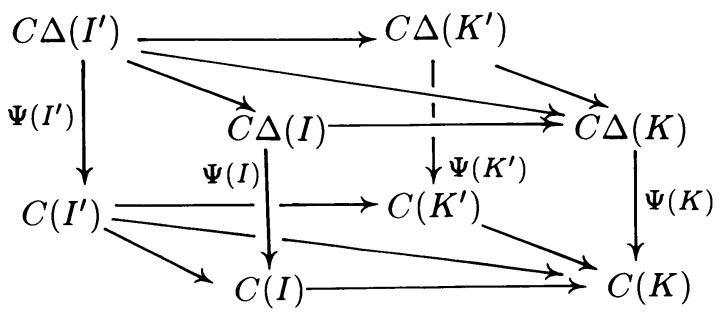

where all of the unlabeled maps are of the form $i(\cdot, \cdot)$. The top and bottom commute by the $\left(I^{\prime}, \pi, J\right)$ and $\left(I^{\prime}, J, \pi\right)$ braid diagrams. The left and right sides commute by the definitions of $\Psi(I)$ and $\Psi(K)$, respectively. The back commutes by induction. Thus, the front commutes on the image of $i: C \Delta\left(I^{\prime}\right) \rightarrow$ $C \Delta(I)$; i.e., the inclusion square commutes on the subspace $C \Delta\left(I^{\prime}\right)$ of $C \Delta(I)$.

To show that if $\pi \in I$, then the inclusion square commutes on $C \Delta(\pi)$, note that $I_{A}=K_{A}$ and $\left(I_{A}, I_{R}, J\right) \in I_{3}(<)$ by Proposition 4.4. The $\left(I_{A}, I_{R}, J\right)$ braid diagram yields $i(I, K) i\left(I_{A}, I\right)=i\left(I_{A}, K\right)$. This and the fact that $I_{A}=K_{A}$ imply $i(I, K) i\left(I_{A}, I\right) p\left(A, I_{A}\right) \tau_{A}=i\left(K_{A}, K\right) p\left(A, K_{A}\right) \tau_{A}$. Therefore $i(I, K) \tau_{I}$ $=\tau_{K}$, implying that $i(I, K) \Psi(I)|C \Delta(\pi)=\Psi(K)| C \Delta(\pi)=\Psi(K) i(I, K) \mid C \Delta(\pi)$. Thus, the inclusion square commutes on the subspace $C \Delta(\pi)$ of $C \Delta(I)$.

It now follows that the inclusion square commutes.

Now consider the projection square. $C \Delta(K)=C \Delta(\pi) \oplus C \Delta\left(K^{\prime}\right)$. We show that the projection square commutes on the subspaces $C \Delta(\pi)$ and $C \Delta\left(K^{\prime}\right)$ of $C \Delta(K)$. We consider two cases: $\pi \in J$ and $\pi \notin J$. 


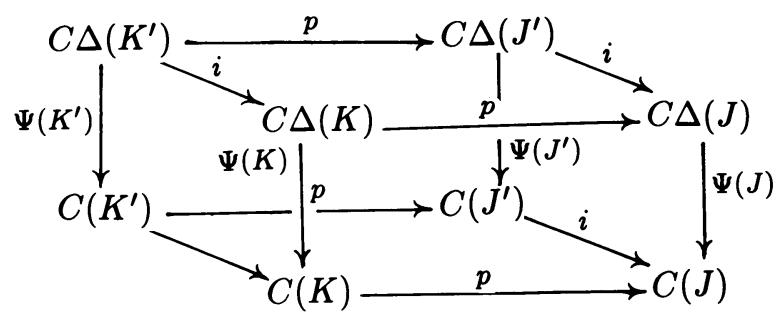

Assume $\pi \in J$. Set $J^{\prime}=J \backslash \pi .\left(I, J^{\prime}, \pi\right) \in I_{3}(<)$. Consider the above diagram.

The top and bottom commute by the $\left(I, J^{\prime}, \pi\right)$ braid diagrams. The left and right sides commute because all inclusion squares commute. The back commutes by induction. Thus the front commutes on the image of $i: C \Delta\left(K^{\prime}\right) \rightarrow$ $C \Delta(K)$; i.e., if $\pi \in J$, then the projection square commutes on the subspace $C \Delta\left(K^{\prime}\right)$ of $C \Delta(K)$.

To show that if $\pi \in J$ then the projection square commutes on $C \Delta(\pi)$, note that

$$
\begin{aligned}
p(K, J) \Psi(K) \mid C \Delta(\pi) & =p(K, J) i\left(K_{A}, K\right) p\left(A, K_{A}\right) \tau_{A} \\
& =i\left(J_{A}, J\right) p\left(A, J_{A}\right) \tau_{A} \\
& =\Psi(J) \mid C \Delta(\pi) \\
& =\Psi(J) p(K, J) \mid C \Delta(\pi)
\end{aligned}
$$

where the second equality holds by Proposition 4.6. It now follows that if $\pi \in J$ then the projection square commutes.

Now assume $\pi \notin J$. Let $I^{\prime}=I \backslash \pi .\left(I^{\prime}, J, \pi\right) \in I_{3}(<)$, and $\pi$ and $J$ are noncomparable. Consider

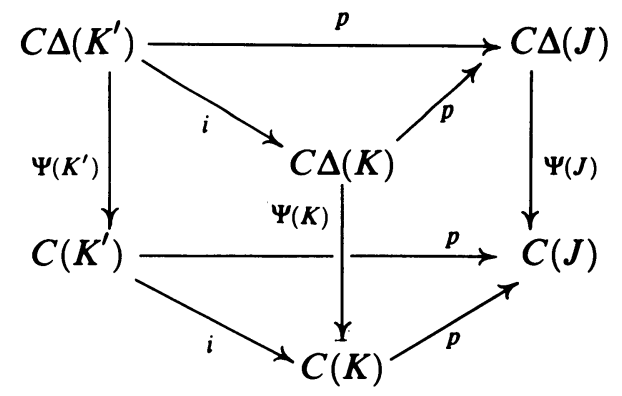

The top and bottom commute by Proposition 4.1. The left side commutes since all inclusion squares commute, and the back side commutes by induction. Therefore the right side commutes on the image of $i: C \Delta\left(K^{\prime}\right) \rightarrow C \Delta(K)$; i.e., the projection square commutes on the subspace $C \Delta\left(K^{\prime}\right)$ of $C \Delta(K)$.

To show that if $\pi \notin J$ then the projection square commutes on $C \Delta(\pi)$, first note that since $\pi \notin J$, it follows that $p(K, J) \mid C \Delta(\pi)=0$. Therefore $\Psi(J) p(K, J) \mid C \Delta(\pi)=0$. It is easy to see that $K_{A} \cap J=\varnothing$. Proposition 4.2 
then implies that

$$
p(K, J) i\left(K_{A}, K\right)=0 .
$$

Therefore $p(K, J) \tau_{K}=p(K, J) i\left(K_{A}, K\right) p\left(A, K_{A}\right) \tau_{A}=0$; i.e.,

$$
p(K, J) \Psi(K) \mid C \Delta(\pi)=0 .
$$

Thus the projection square commutes on $C \Delta(\pi)$, and it now follows that diagram (4.7) commutes.

The proof of Theorem 4.8 is complete once we show that $\Psi(K)$ is a chain map. Denote the boundary map in the chain complex $C(K)$ by $\partial(K)$. We show that $\partial(K) \Psi(K)=\Psi(K) \Delta(K)$ on each of the subspaces $C \Delta\left(K^{\prime}\right)$ and $C \Delta(\pi)$ of $C \Delta(K)$. Note that $\Psi(A)$ is a chain map by Proposition 4.7.

Now note that

$$
\begin{aligned}
\partial(K) \Psi(K) \mid C \Delta\left(K^{\prime}\right) & =\partial(K) i\left(K^{\prime}, K\right) \Psi\left(K^{\prime}\right) \\
& =i\left(K^{\prime}, K\right) \Psi\left(K^{\prime}\right) \Delta\left(K^{\prime}\right) \\
& =\left(\Psi(K) \mid C \Delta\left(K^{\prime}\right)\right) \Delta\left(K^{\prime}\right) \\
& =\Psi(K) \Delta(K) \mid C \Delta\left(K^{\prime}\right),
\end{aligned}
$$

where the first and third equalities hold by the definition of $\Psi(K)$, and the second holds since $i\left(K^{\prime}, K\right)$ and $\Psi\left(K^{\prime}\right)$ are chain maps. Furthermore, note that

$$
\begin{aligned}
\partial(K) \Psi(K) \mid C \Delta(\pi) & =\partial(K) i\left(K_{A}, K\right) p\left(A, K_{A}\right) \Psi(A) \mid C \Delta(\pi) \\
& =i\left(K_{A}, K\right) p\left(A, K_{A}\right) \Psi(A) \Delta(A) \mid C \Delta(\pi) \\
& =\Psi(K) i\left(K_{A}, K\right) P\left(A, K_{A}\right) \Delta(A) \mid C \Delta(\pi) \\
& =\Psi(K) \Delta(K) i\left(K_{A}, K\right) P\left(A, K_{A}\right) \mid C \Delta(\pi) \\
& =\Psi(K) \Delta(K) \mid C \Delta(\pi),
\end{aligned}
$$

where the first equality holds by the definition of $\Psi(K)$, the second and fourth hold since $i, p$, and $\Psi(A)$ are chain maps, and the third holds by the commutativity of diagram (4.7). Therefore $\Psi(K)$ is a chain map, and the proof of Theorem 4.8 is complete.

\section{THE CONNECTION MATRIX THEORY FOR MORSE DECOMPOSITIONS}

Recall that $M=\{M(\pi)\}_{\pi \in P}$ is a Morse decomposition of the isolated invariant set $S$ with admissible ordering $<$, and $\mathscr{H}(<; G) \quad(=\mathscr{H}(<))$ is the homology index braid of < with coefficients in $G . \mathscr{H}(<)$ is chain complex generated; therefore if $C=\{C \Delta(\pi)\}_{\pi \in P}$ is a collection of free chain complexes such that the homology of $C \Delta(\pi)$ is isomorphic to $H(\pi)$, the homology index of $M(\pi)$ with coefficients in $G$, then $\mathscr{C} \mathscr{M}(\mathscr{H}(<), C)$, the collection of $C$-connection matrices of $\mathscr{H}(<)$, is nonempty.

Definition 5.1. Under the above circumstances

A. $\mathscr{C H}(<), C)$ is called the $C$-connection matrices of the admissible ordering < with coefficients in $G$, and is denoted $\mathscr{C} \mathscr{M}(<; G, C)$. 
B. If $<$ is the flow ordering, then $\mathscr{C} \mathscr{M}(\mathscr{H}(<), C)$ is called the $C$-connection matrices of the Morse decomposition $M$ with coefficients in $G$, and is denoted $\mathscr{E} \mathbb{M}(M ; G, C)$.

C. If the chain complex $C \Delta(\pi)$ equals $H(\pi)$ with trivial boundary map, then the above collections are denoted $\mathscr{C} \mathscr{M}(<; G)$ and $\mathscr{\mathscr { M }}(M ; G)$, and are called the connection matrices of $<$ and $M$, respectively, with coefficients in $G$.

Note that the situation described in Definition 5.1.C occurs when the homology indices $H(\pi)$ are free for each $\pi$. An example of that case is when the coefficient module is chosen to be a field (see Examples 6.1-6.3 below).

Since every admissible ordering of $M$ is an extension of the flow ordering, the following proposition is an immediate consequence of Proposition 3.7.

Proposition 5.2. $\mathscr{C} \mathscr{M}(M ; G, C) \subset \mathscr{C} \mathscr{M}(<; G, C)$.

Proposition 5.2 is a reflection of the fact that the connection matrices of the flow ordering of the Morse decomposition are defined using maximum (over all other admissible orderings of the Morse decomposition) algebraic information.

As is indicated in $\S 2$, it is expected that the connection matrices provide some information about the structure of the invariant set $S$. For an initial interpretation result we have

Proposition 5.3. If $\Delta \in \mathscr{C} \mathscr{M}(M ; G, C), \pi$ and $\pi^{\prime}$ are adjacent in the flow ordering, and $\Delta\left(\pi^{\prime}, \pi\right) \neq 0$, then $C\left(M\left(\pi^{\prime}\right), M(\pi)\right) \neq \varnothing$.

Proposition 5.3 describes a situation where information about the set of connecting orbits between elements of a Morse decomposition can be obtained via the connection matrices of the Morse decomposition. We point out that the proof is nothing but a simple consequence of the definitions involved; specifically,

Proof. $\Delta\left(\pi^{\prime}, \pi\right) \neq 0$ implies $\pi<_{F} \pi^{\prime}$. Therefore by the definition of the flow ordering there is a sequence of distinct elements of $P: \pi=\pi_{0}, \ldots, \pi_{n}=\pi^{\prime}$ with $C\left(M\left(\pi_{j}\right), M\left(\pi_{j-1}\right)\right) \neq \varnothing$ for each $j=1, \ldots, n$. $\pi$ and $\pi^{\prime}$ adjacent in the flow ordering then implies $n=1$; i.e., $C\left(M\left(\pi^{\prime}\right), M(\pi)\right) \neq \varnothing$.

The ease with which Proposition 5.3 follows from the relevant definitions is significant because it indicates that the object under investigation, i.e., the collection of connection matrices of the flow ordering of a Morse decomposition, is to some degree properly defined as a tool for studying the structure of the invariant set relative to the Morse decomposition. We leave further interpretation results for future investigations; however, it should be noted that simple examples do indicate that deeper interpretation results are possible.

For example, suppose that for some strictly upper triangular connection matrix of the Morse decomposition, the composition $\Delta\left(\pi^{\prime}, \pi\right) \Delta\left(\pi^{\prime \prime}, \pi^{\prime}\right)$ is nontrivial and that $\pi$ and $\pi^{\prime}$ as well as $\pi^{\prime}$ and $\pi^{\prime \prime}$ are adjacent under the flow ordering. Then by Proposition 5.3 both $C\left(M\left(\pi^{\prime \prime}\right), M\left(\pi^{\prime}\right)\right)$ and $C\left(M\left(\pi^{\prime}\right), M(\pi)\right)$ are nonempty. Furthermore, if $\Pi:=\left\{\pi, \pi^{\prime}, \pi^{\prime \prime}\right\}$, then since $\Delta(\Pi)^{2} \neq 0$, it 


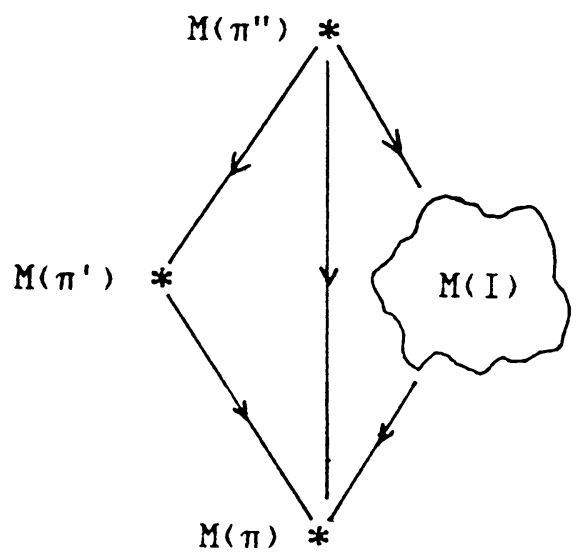

FIGURE 5.1

follows (by Proposition 3.2) that $\Pi$ is not an interval of the flow ordering, thus implying that more structure is present. More specifically, it is not difficult to see that this implies $C\left(M\left(\pi^{\prime \prime}\right), M(\pi)\right) \neq \varnothing$, and moreover that there exists an interval $I$ in the flow ordering such that $\pi \notin I$ and both $C\left(M\left(\pi^{\prime \prime}\right), M(I)\right)$ and $C(M(I), M(\pi))$ are nonempty (see Figure 5.1).

The schematic representation of this situation suggests that there is some further structure to $C\left(M\left(\pi^{\prime \prime}\right), M(\pi)\right.$ ) (e.g., a parameterized family of orbits). As is indicated above, we leave a formal presentation of these points, along with further connection matrix interpretation results, to a later study.

\section{SOME EXAMPLES}

To begin, consider the following family of ordinary differential equations parameterized by the variable $\theta>0$ :

$$
\dot{x}=+y, \quad \dot{y}=+\theta y-x\left(x-\frac{1}{3}\right)(1-x) .
$$

The complete set of bounded solutions $S_{\theta}$ for these equations is shown (along with some nearby orbits) for values of $\theta$ near 0 and for values of $\theta$ large in Figure 6.1. For all $\theta>0$ the set of bounded solutions is an isolated invariant set, and the collection $M_{\theta}=\left\{M_{\theta}(i)\right\}$ is a Morse decomposition of $S_{\theta}$ where the sets $M_{\theta}(1), M_{\theta}(2), M_{\theta}(3)$ are the points in the $x y$ plane $(1 / 3,0),(0,0)$, $(1,0)$, respectively.

In Example 6.1 we compute the connection matrices with coefficients in $\mathbf{Z}_{2}$ of the Morse decompositions depicted in Figure 6.1. In Example 6.2 we use the connection matrix theory to prove that there exists a parameter value $\theta^{*}$ at which there is a connecting orbit from $M_{\theta^{*}}$ (3) to $M_{\theta^{*}}(2)$. In Example 6.3 we compute the connection matrices with coefficients in $\mathbf{Z}_{2}$ for the Morse decomposition $M_{\theta}$. . The latter example is an instance where the Morse decomposition does not have a unique connection matrix.

Note that since we have chosen field coefficients in Examples 6.1-6.3 and since we are computing connection matrices (i.e., $C$-connection matrices where 


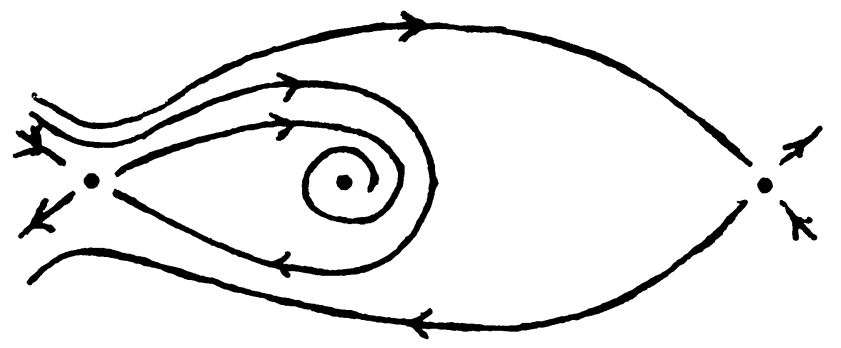

$\theta \gg 1$

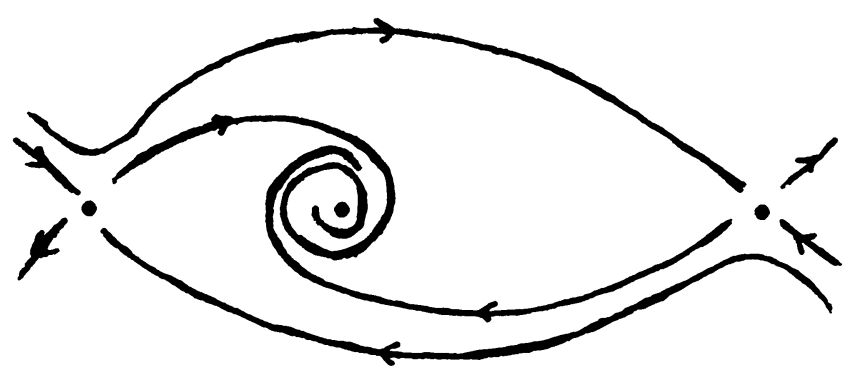

$M_{\theta}(2) \quad M_{\theta}(1) \quad M_{\theta}(3)$

FIGURE 6.1

$C$ is the collection of homology indices of the sets in the relevant Morse decomposition), it follows that the connection matrices are strictly upper triangular (see the remarks preceding Definition 3.6 and following Definition 5.1).

In Example 6.4 we consider a case where the coefficients are chosen to be in $\mathbb{Z}$ (i.e., not in a field) and there are no connection matrices. However, with an appropriate choice of $C$, we compute the $C$-connection matrices.

Example 6.1. Qualitatively the flows in Figure 6.1 can be depicted as in Figure 6.2 below.

Consider the case where $0<\theta \ll 1$. Here the flow ordering is such that $1<_{F} 2$ is the only relation. Thus by strict upper triangularity of the connection

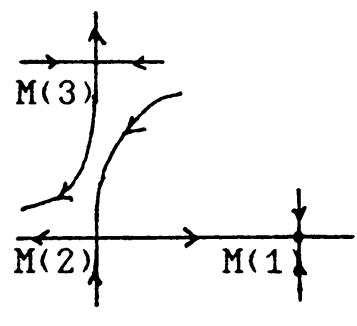

$0<\theta \ll 1$

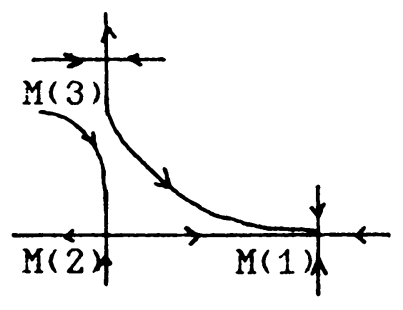

$\theta \gg 1$ 
matrices it follows that only $\Delta(2,1)$ may be nonzero. The homology index of the Morse set $M(12)$ is easily seen to be trivial; therefore $H \Delta(12)$ must be also. Since the homology indices of the Morse sets $M(1)$ and $M(2)$ are nontrivial, it follows that $\Delta(2,1)$ is an isomorphism. Thus $\mathscr{\mathscr { M }}\left(M_{\theta} ; \mathbb{Z}_{2}\right)$ consists of one matrix, and it is in the form

$$
\left(\begin{array}{ccc}
0 & \approx & 0 \\
0 & 0 & 0 \\
0 & 0 & 0
\end{array}\right)
$$

where $\approx$ indicates an entry that is an isomorphism.

Consider the case where $\theta \gg 1$. Here the flow ordering is such that $1<_{F} 2$ and $1<_{F} 3$ are the only relations. Thus by strict upper triangularity of the connection matrices it follows that only $\Delta(2,1)$ and $\Delta(3,1)$ may be nonzero, and furthermore, by an argument identical to the one used above, it follows that each of these entries is an isomorphism. Thus in this case $\mathscr{C} \mathbb{M}\left(M_{\theta} ; \mathbb{Z}_{2}\right)$ also contains a single matrix, and it is in the form

$$
\left(\begin{array}{ccc}
0 & \approx & \approx \\
0 & 0 & 0 \\
0 & 0 & 0
\end{array}\right) .
$$

Example 6.2. Now let $0<\theta^{\prime} \ll 1$ and $1 \ll \theta^{\prime \prime}$. Append the equation

$$
\dot{\theta}=\varepsilon\left(\theta^{\prime}-\theta\right)\left(\theta^{\prime \prime}-\theta\right), \quad \varepsilon>0,
$$

to the above system. $S$, the complete set of bounded solutions to the resulting system, is an isolated invariant set. Some of the orbits in $S$, along with a Morse decomposition, $M=\left\{M\left(i^{\prime}\right), M\left(i^{\prime \prime}\right)\right\}$, of $S$, are shown in Figure 6.3.

It is not difficult to see that if $\Delta$ is a connection matrix of $M$ with coefficients in $\mathbb{Z}_{2}$ then $\Delta=\left(\begin{array}{cc}\Delta^{\prime} & T \\ 0 & \Delta^{\prime \prime}\end{array}\right)$ where $\Delta^{\prime}$ and $\Delta^{\prime \prime}$ are of the form of the first and second matrices, respectively, computed in Example 6.1 and the entries in $T$ are in the form $\Delta\left(i^{\prime \prime}, j^{\prime}\right)$. The matrix $T$ is called a transition matrix of the system; transition matrices are studied further in [11-13]. We are interested in

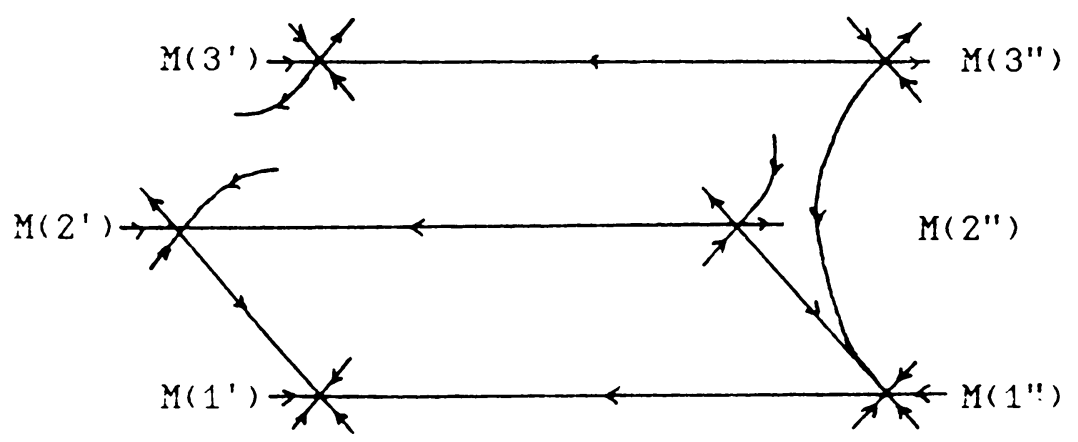

Figure 6.3 
the $\Delta\left(3^{\prime \prime}, 2^{\prime}\right)$ entry. It is easily verified that $\Delta\left(1^{\prime \prime}, 1^{\prime}\right)$ is an isomorphism, and therefore

$$
\Delta=\left(\begin{array}{cccccc}
0 & \approx & 0 & \approx & \bullet & \bullet \\
0 & 0 & 0 & \bullet & \bullet & \Delta\left(3^{\prime \prime}, 2^{\prime}\right) \\
0 & 0 & 0 & \bullet & \bullet & \bullet \\
0 & 0 & 0 & 0 & \approx & \approx \\
0 & 0 & 0 & 0 & 0 & 0 \\
0 & 0 & 0 & 0 & 0 & 0
\end{array}\right)
$$

$\Delta^{2}$ must be 0 ; therefore it can be seen by composing the top row with the right hand column that $\Delta\left(3^{\prime \prime}, 2^{\prime}\right)$ is nontrivial. Then since $M\left(3^{\prime \prime}\right)$ and $M\left(2^{\prime}\right)$ are adjacent in the flow ordering, it follows that $C\left(M\left(3^{\prime \prime}\right), M\left(2^{\prime}\right)\right)$ is nonempty. Note that this is true for all $\varepsilon>0$. It then follows (see [12]) that in the system at $\varepsilon=0$ (i.e., in the original parameterized family of equations) there exists $\theta^{*} \in\left(\theta^{\prime}, \theta^{\prime \prime}\right)$ such that $C\left(M_{\theta^{*}}(3), M_{\theta^{*}}(2)\right)$ is nonempty.

An alternative proof of the existence of a parameter value $\theta^{*}$ for which there is a connecting orbit from $M_{\theta^{*}}(3)$ to $M_{\theta^{*}}$ (2) is presented using the connection matrix continuation theory in [5].

Example 6.3. Now consider the flow at $\theta^{*}$; it is depicted qualitatively along with an index filtration for the flow ordering of the Morse decomposition in Figure 6.4 below. Let $H(I)$ denote the homology index with coefficients in $\mathbb{Z}_{2}$ of the Morse set $M(I)$ for each $I \in I\left(<_{F}\right)$. Each homology index can be computed by choosing appropriate index pairs from the index filtration (for example, see the schematic representations in Figure 6.5 below).

The relations in the flow ordering are $1<_{F} 2,2<_{F} 3$, and $1<_{F} 3$. Thus if $\Delta$ is a connection matrix of the Morse decomposition, then only $\Delta(2,1)$, $\Delta(3,2)$, and $\Delta(3,1)$ may be nontrivial. As in Example $6.1, \Delta(2,1)$ is an isomorphism. $\Delta(3,2)$ is trivial because $H(3)$ is nontrivial only in dimension one, $\Delta(3,2)$ is of degree -1 , and $H(2)$ is trivial in dimension 0 .

Now consider $\Delta(3,1)$. We leave the details of the computation to the reader (see [3]), and only illustrate the algebraic relations that determine $\Delta(3,1)$. Let

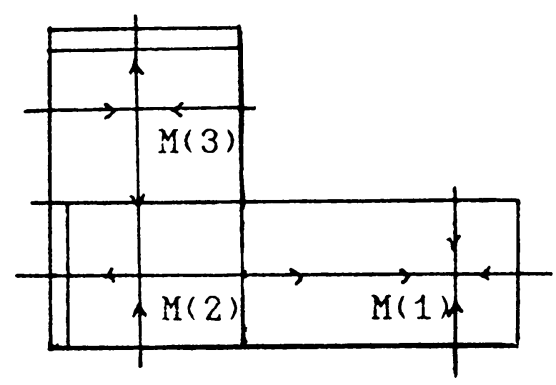

FIGURE 6.4 


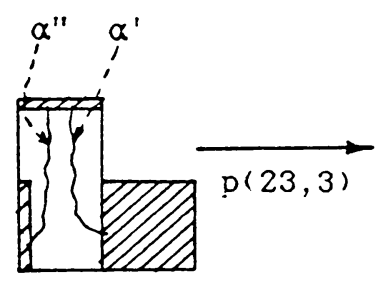

$\mathrm{H}(23)$

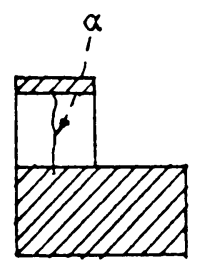

$\mathrm{H}(3)$

A

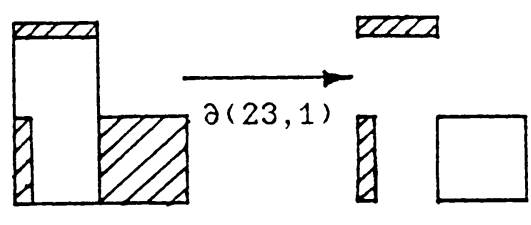

$H(23)$

B

FIGURE 6.5

$\alpha$ be a generator of $H(3)$, and note that the map $p(23,3): H(23) \rightarrow H(3)$ associates two independent generators $\left(\alpha^{\prime}\right.$ and $\alpha^{\prime \prime}$ in Figure 6.5.A) of $H(23)$ to $\alpha$. It is via this association that $\Delta(3,1) \alpha$ is defined.

The flow-defined boundary map $\partial(23,1): H(23) \rightarrow H(1)$ is pictured schematically in Figure 6.5.B. It is easy to see that $\partial(23,1) \alpha^{\prime}$ is a generator of $H(1)$, but $\partial(23,1) \alpha^{\prime \prime}$ is trivial. Thus, depending whether $\alpha$ is associated to $\alpha^{\prime}$ or $\alpha^{\prime \prime}, \Delta(3,1)$ is either an isomorphism or is trivial. It follows that $\mathscr{C} \mathscr{M}\left(M_{\theta}, ; \mathbb{Z}_{2}\right)$ contains two matrices, and they are of the form

$$
\left(\begin{array}{ccc}
0 & \approx & 0 \\
0 & 0 & 0 \\
0 & 0 & 0
\end{array}\right) \text { and }\left(\begin{array}{ccc}
0 & \approx & \approx \\
0 & 0 & 0 \\
0 & 0 & 0
\end{array}\right) .
$$

In this example the nonuniqueness of the connection matrices reflects the fact that there are two qualitatively different perturbations possible from the flow at $\theta^{*}$. It is not known if nonuniqueness of connection matrices in general reflects such a bifurcation of behavior. Reineck [12] has shown that on a smooth manifold if the Morse decomposition consists of hyperbolic rest points and is such that stable and unstable manifolds intersect transversally then the connection matrix is unique. Further results in that direction would be an important component of the connection matrix interpretation theory.

Note that $\mathscr{C} \mathscr{M}\left(M_{\theta^{*}} ; \mathbb{Z}_{2}\right)$ does not continue to $\mathscr{\mathscr { C }}\left(M_{\theta} ; \mathbb{Z}_{2}\right)$ for $\theta$ near $\theta^{*}$ (i.e., the form of the collection changes). Thus, even though the Morse decomposition continues locally, the collection of connection matrices is not invariant under continuation. However, as this example illustrates, the connection matrices are upper semicontinuous in the sense that under local continuation connection matrices may be lost, but not gained (see [5]). 
Example 6.4. Now consider the flow on the Mobius band $S$ pictured in Figure 6.6 below:

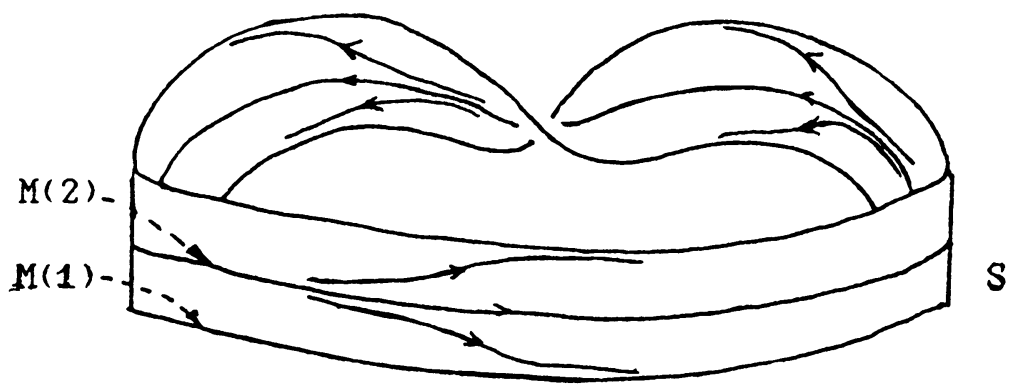

FIGURE 6.6

$M(1)$ is an attracting periodic orbit, and $M(2)$ is the complementary repelling orbit. Let $H(\cdot)$ denote the homology index with coefficients in $\mathbf{Z}$ of the relevant Morse set of the flow ordering of the above Morse decomposition. The associated homology index braid consists of the sequence of graded Z-modules and maps

$$
\cdots \rightarrow H(2) \stackrel{\partial}{\longrightarrow} H(1) \stackrel{i}{\longrightarrow} H(12) \stackrel{p}{\longrightarrow} H(2) \stackrel{\partial}{\longrightarrow} H(1) \rightarrow \cdots,
$$

where the only nontrivial modules occur in dimension 1, and appear as follows:

$$
\cdots \rightarrow 0 \rightarrow \mathbf{Z} \stackrel{\times 2}{\longrightarrow} \mathbf{Z} \stackrel{p}{\longrightarrow} \mathbf{Z}_{2} \rightarrow 0 \rightarrow \cdots .
$$

There are no connection matrices associated to this graded module braid (see the remarks preceding Definition 3.6); however, with an appropriate choice of the collection $C$, the collection of $C$-connection matrices is nonempty. To that end let $C \Delta(1)$ be a chain complex with graded module equal to $H(1)$ and with trivial boundary map, and let $C \Delta(2)$ be a chain complex with graded module $\mathbf{Z}$ in dimensions 1 and 2 , trivial otherwise, and with chain map $\Delta(2)$ multiplication by 2 in dimension 2, trivial otherwise. Let $C=\{C \Delta(1), C \Delta(2)\}$; then since $C \Delta(1)$ and $C \Delta(2)$ are free chain complexes having homology isomorphic to $H(1)$ and $H(2)$, respectively, it follows that the collection of $C$-connection matrices of the Morse decomposition is nonempty. In fact, it contains a single matrix $\left(\begin{array}{cc}0 & \Delta(2,1) \\ 0 & \Delta(2)\end{array}\right)$ where $\Delta(2)$ is the map described above and $\Delta(2,1)$ is an isomorphism in dimension 2 and trivial otherwise.

Note that the flow-defined boundary map $\partial(2,1)$ is trivial and therefore does not reflect the existence of a connecting orbit from $M(2)$ to $M(1)$; however $\Delta(2,1)$ is nontrivial, and therefore (by Proposition 5.3) $C(M(2), M(1)) \neq \varnothing$.

These examples provide simple illustrations of applications of the connection matrix to the qualitative study of differential equations. Studies of particular differential equations using the connection matrix have been carried out by Mischaikow [11] and Reineck [12]. These examples also illustrate that there 
are many questions to be answered (and asked) regarding the connection matrix theory. Besides the interpretation questions mentioned above, there are numerous questions regarding the computation of connection matrices, most importantly those investigating the minimal algebraic information required to maximize the information in the connection matrix.

\section{ACKNOWLEDGEMENT}

Charles Conley, my advisor at the University of Wisconsin, planted the seed out of which this theory has grown. My indebtedness to him for his insights and inspiration goes without saying. He is missed, but his influence will long live on.

\section{REFERENCES}

1. C. Conley, Isolated invariant sets and the Morse index, CBMS Regional Conf. Ser. in Math., no. 38, Amer. Math. Soc., Providence, R.I., 1980.

2. C. Conley and E. Zehnder, Morse-type index theory for flows and periodic solutions for Hamiltonian equations, Comm. Pure Appl. Math. 37 (1984).

3. R. Franzosa, Index filtrations and connection matrices for partially ordered Morse decompositions, Ph. D. dissertation, Univ. of Wisconsin-Madison, 1984.

4. __ Index filtrations and the homology index braid for partially ordered Morse decompositions, Trans. Amer. Math. Soc. 298 (1986).

5. _ The continuation theory for Morse decompositions and connection matrices, Trans. Amer. Math. Soc. 310 (1988).

6. R. Franzosa and K. Mischaikow, The connection matrix theory for semiflows on (not necessarily locally compact) metric spaces, J. Differential Equations 71 (1988).

7. H. Kurland, The Morse index of an isolated invariant set is a connected simple system, J. Differential Equations 42 (1981).

8. __ Homotopy invariants of repeller-attractor pairs. I, The Püppe sequence of an $r$-a pair, J. Differential Equations 46 (1982).

9. __ Homotopy invariants of repeller-attractor pairs. II, Continuation of $r$-a pairs, J. Differential Equations 49 (1983).

10. C. McCord, Mappings and homological properties in the Conley index theory, $\mathrm{Ph}$. D. dissertation, Univ. of Wisconsin-Madison, 1986.

11. K. Mischaikow, Classification of traveling wave solutions of reaction-diffusion systems, Brown Univ., LCDS \#86-5, 1985.

12. J. Reineck, The connection matrix and the classification of flows arising from ecological models, Ph. D. dissertation, Univ. of Wisconsin-Madison, 1985.

13. __ Connecting orbits in one parameter families of flows, preprint.

14. D. Salamon, Connected simple systems and the Conley index of isolated invariant sets, Trans. Amer. Math. Soc. 291 (1985).

15. J. Smoller, Shock waves and reaction-diffusion equations, Springer-Verlag, Berlin and New York, 1983.

16. E. Spanier, Algebraic topology, McGraw-Hill, New York, 1966.

Department of Mathematics, University of Maine, Orono, Maine 04469 